Supporting Information

\title{
CO Adsorbate Promotes Polaron Photoactivity on the Reduced Rutile $\mathrm{TiO}_{2}(110)$ Surface
}

\author{
Cheng Cheng, ${ }^{1}$ Yonghao Zhu, ${ }^{1}$ Wei-Hai Fang, ${ }^{1}$ Run Long, ${ }^{1 *}$ Oleg V. Prezhdo ${ }^{2}$ \\ ${ }^{1}$ College of Chemistry, Key Laboratory of Theoretical \& Computational Photochemistry of \\ Ministry of Education, Beijing Normal University, Beijing, 100875, People's Republic of \\ China \\ ${ }^{2}$ Department of Chemistry, University of Southern California, Los Angeles, California 90089, \\ United States
}

* Corresponding Author Email: runlong@bnu.edu.cn 


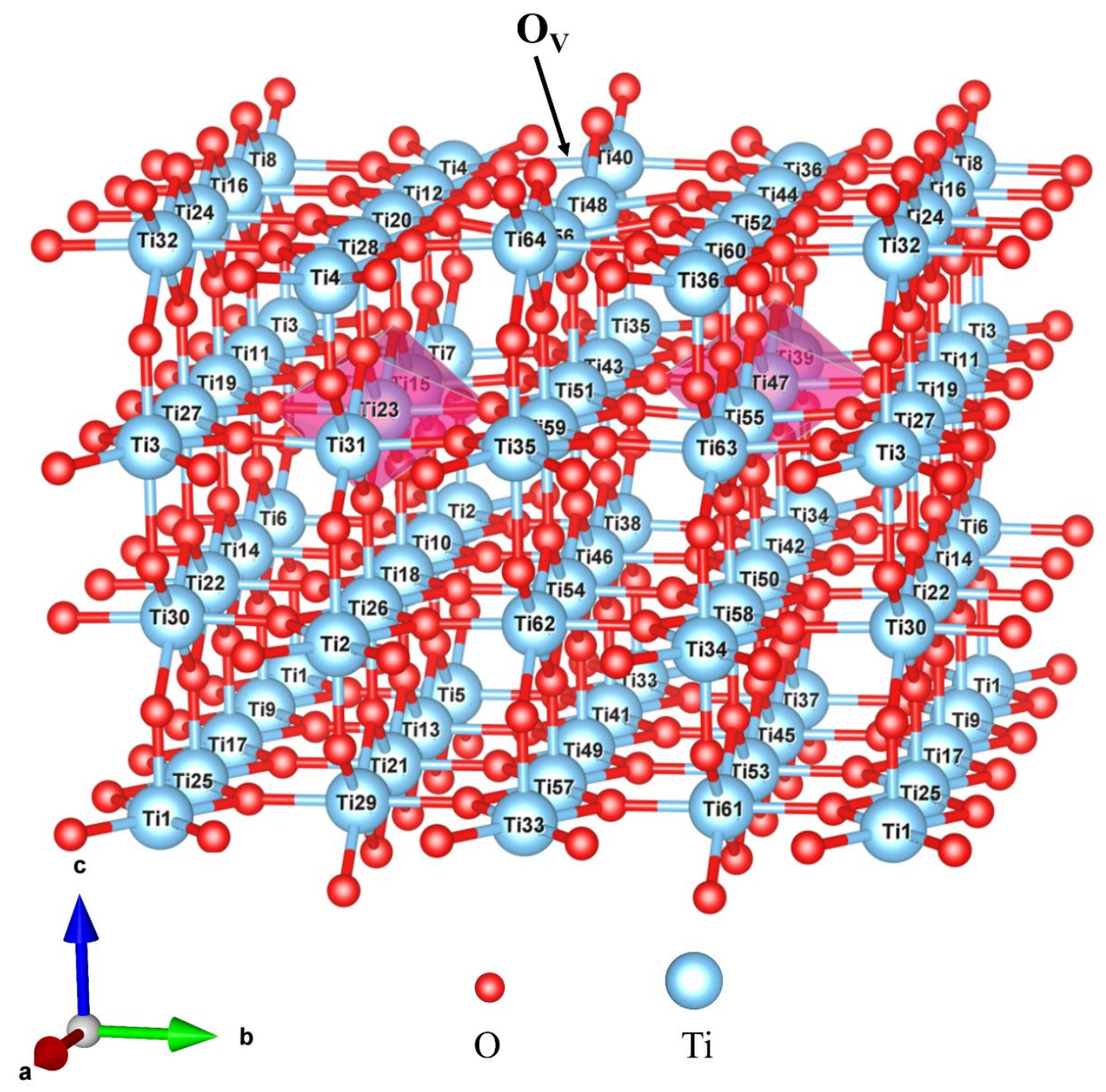

Figure S1. Ball and stick model of the defective $\mathrm{TiO}_{2}(110)$ surface with two excess electrons. The numerical labels at $\mathrm{Ti}$ atoms help to identify the different localizations of the $\mathrm{O}_{\mathrm{v}}$-induced excess charge. 


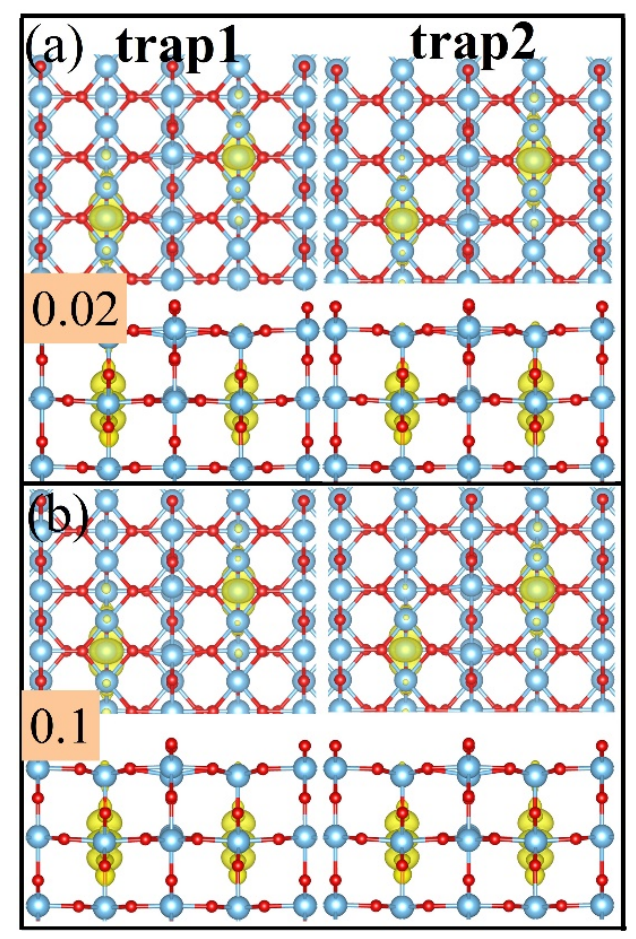

Figure S2. Charge densities of the polaron states for the $\mathrm{O}_{\mathrm{V}} / \mathrm{TiO}_{2}$ system calculated using the $\mathrm{PBE}+\mathrm{U}$ method with different electron smearing: (a) $0.02 \mathrm{eV}$ and (b) $0.1 \mathrm{eV}$. The corresponding plots for the $0.05 \mathrm{eV}$ smearing are shown in the top panel of Figure S4. The charge densities obtained with the HSE06 functional are plotted in Figure 2. There are no discernable differences between the densities obtained using the different smearing values and functionals. 


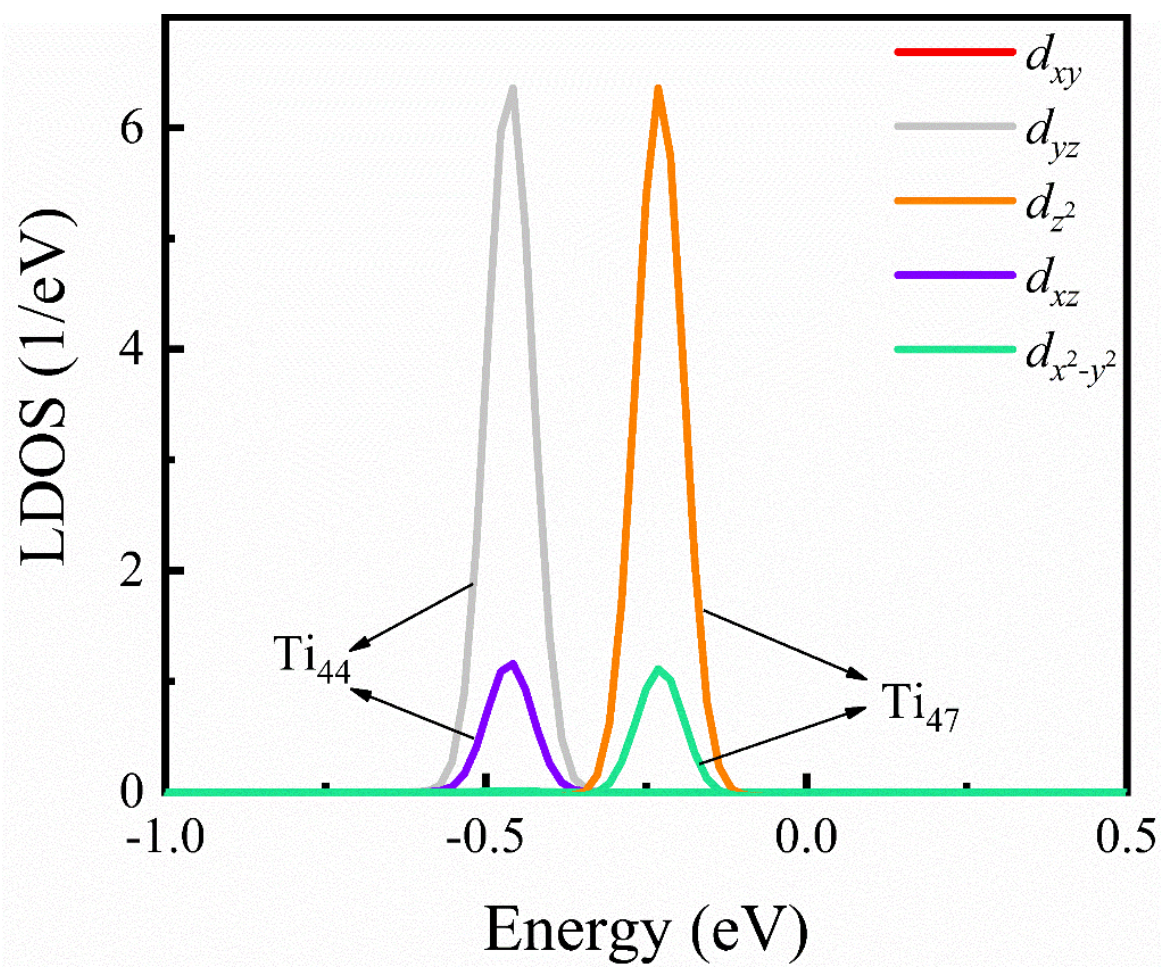

Figure S3. Localized density of states (LDOS) showing $d$-orbital character of the Ti electron polarons in the $\mathrm{O}_{\mathrm{V}} / \mathrm{TiO}_{2}$ and $\mathrm{CO} / \mathrm{TiO}_{2}$ systems. $\mathrm{Ti}_{47}$ and $\mathrm{Ti}_{44}$ denote the special $\mathrm{Ti}$ atoms in the $\mathrm{O}_{\mathrm{v}} / \mathrm{TiO}_{2}$ and $\mathrm{CO} / \mathrm{TiO}_{2}$ systems labeled in Figures $\mathrm{S} 1$ and $\mathrm{S} 2$, respectively. The electron polaron in the subsurface ( $\mathrm{Ti}_{47}$ ) shows the $d_{z}^{2}$ symmetry and a smaller $d_{z^{2}-y^{2}}$ contribution. In comparison, the electron polaron in the surface $\left(\mathrm{Ti}_{44}\right)$, created by interaction with the $\mathrm{CO}$ molecule, shows the $d_{y z}$ symmetry together with a smaller $d_{x z}$ contribution. The results demonstrate that CO alters the orbital character of the electron polaron significantly, shifting it from the $d_{z}^{2}$ orbital to the lower-energy $d_{y z}$ orbital. 


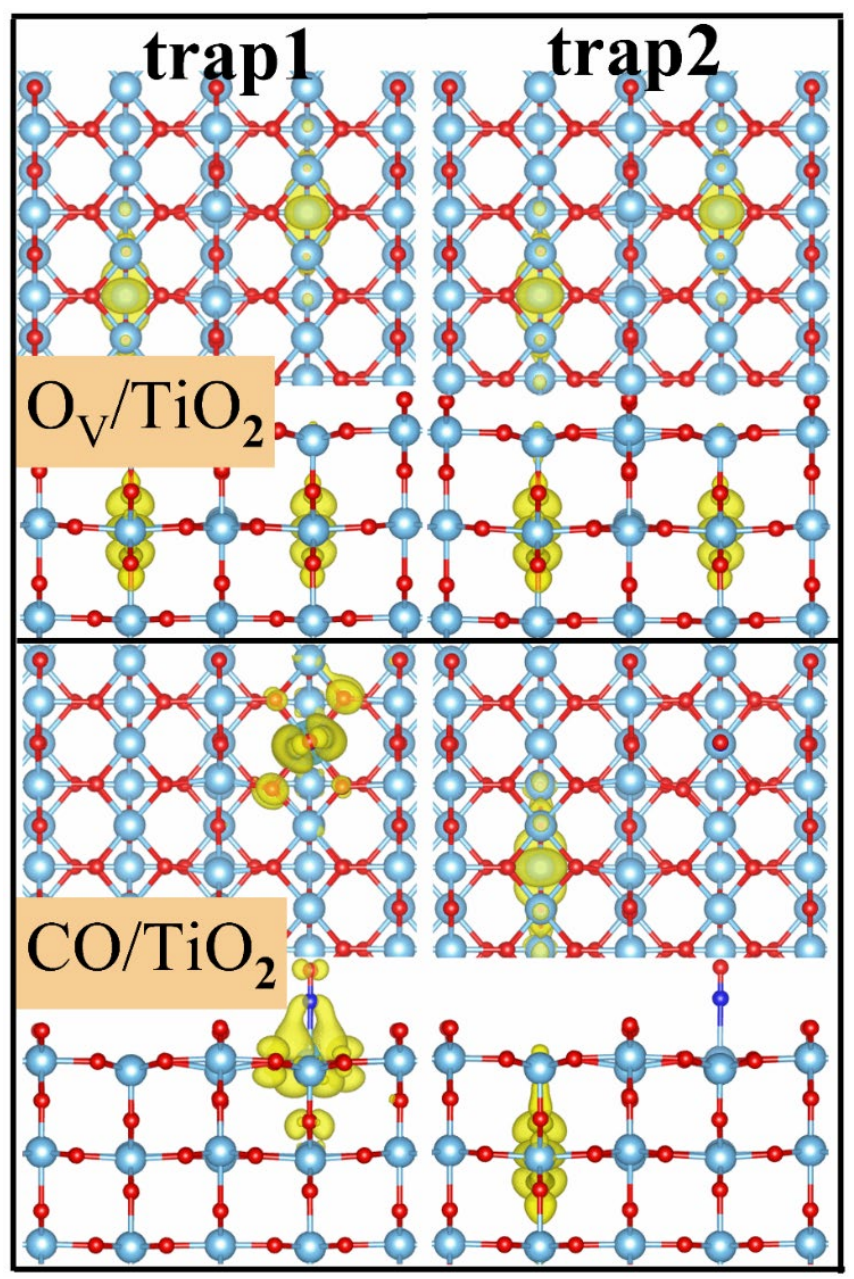

Figure S4. Charge densities of the polaron states in the $\mathrm{Ov} / \mathrm{TiO}_{2}$ and $\mathrm{CO} / \mathrm{TiO}_{2}$ systems calculated using the PBE+U method with the $0.05 \mathrm{eV}$ smearing. This method is used for the majority of the calculations. There are no obvious differences with the densities obtained using the HSE06 hybrid functional, Figure 2, and PBE+U with different smearing, Figure S2. 

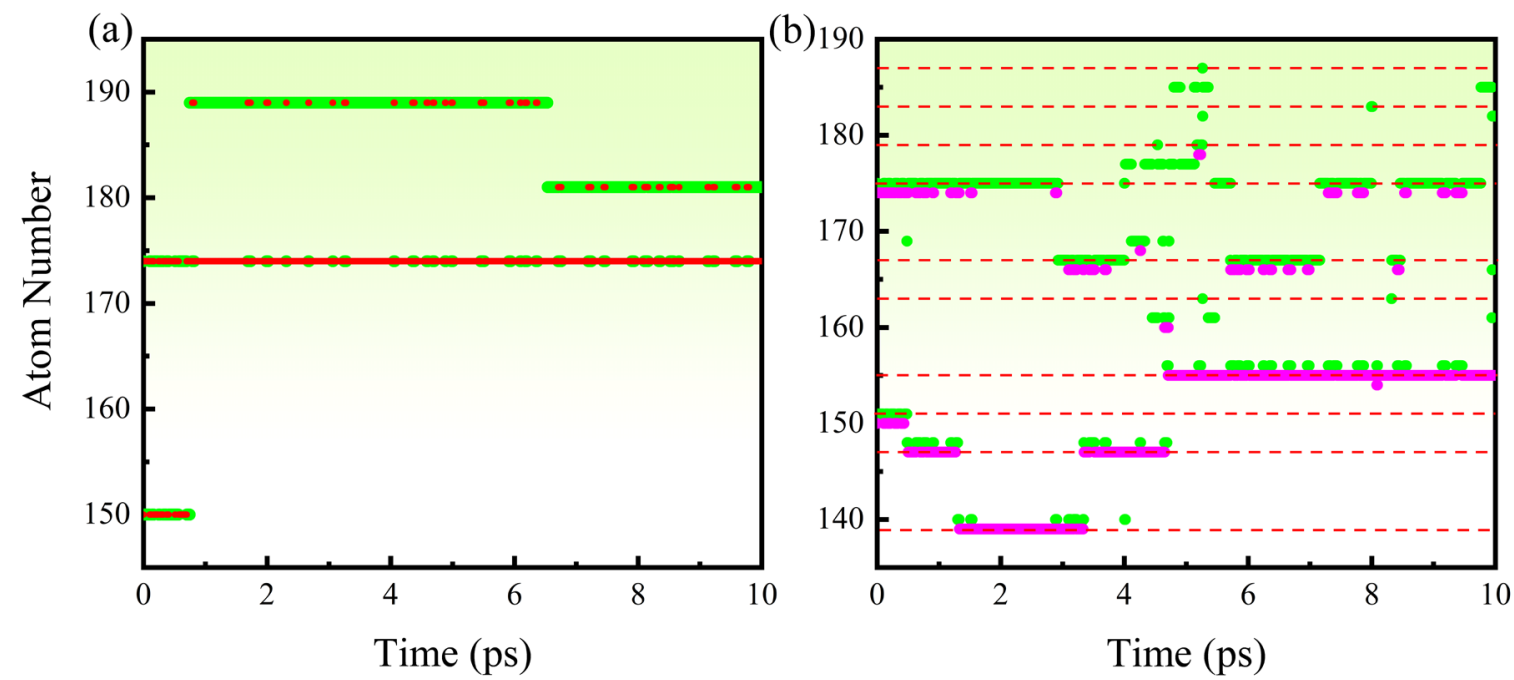

Figure S5. Polaron positions at every MD step during the $10 \mathrm{ps} P B E+U$ simulations carried out at (a) $300 \mathrm{~K}$ and (b) $700 \mathrm{~K}$ in $\mathrm{O}_{\mathrm{V}} / \mathrm{TiO}_{2}$. The red dashed lines denote positions of the surface atoms supporting surface polarons. The atom numbers are provided with the atom coordinates at the end of this file. The electron polarons induced by the oxygen vacancy hop frequently to surface sites at $700 \mathrm{~K}$ and less frequently at $300 \mathrm{~K}$. 


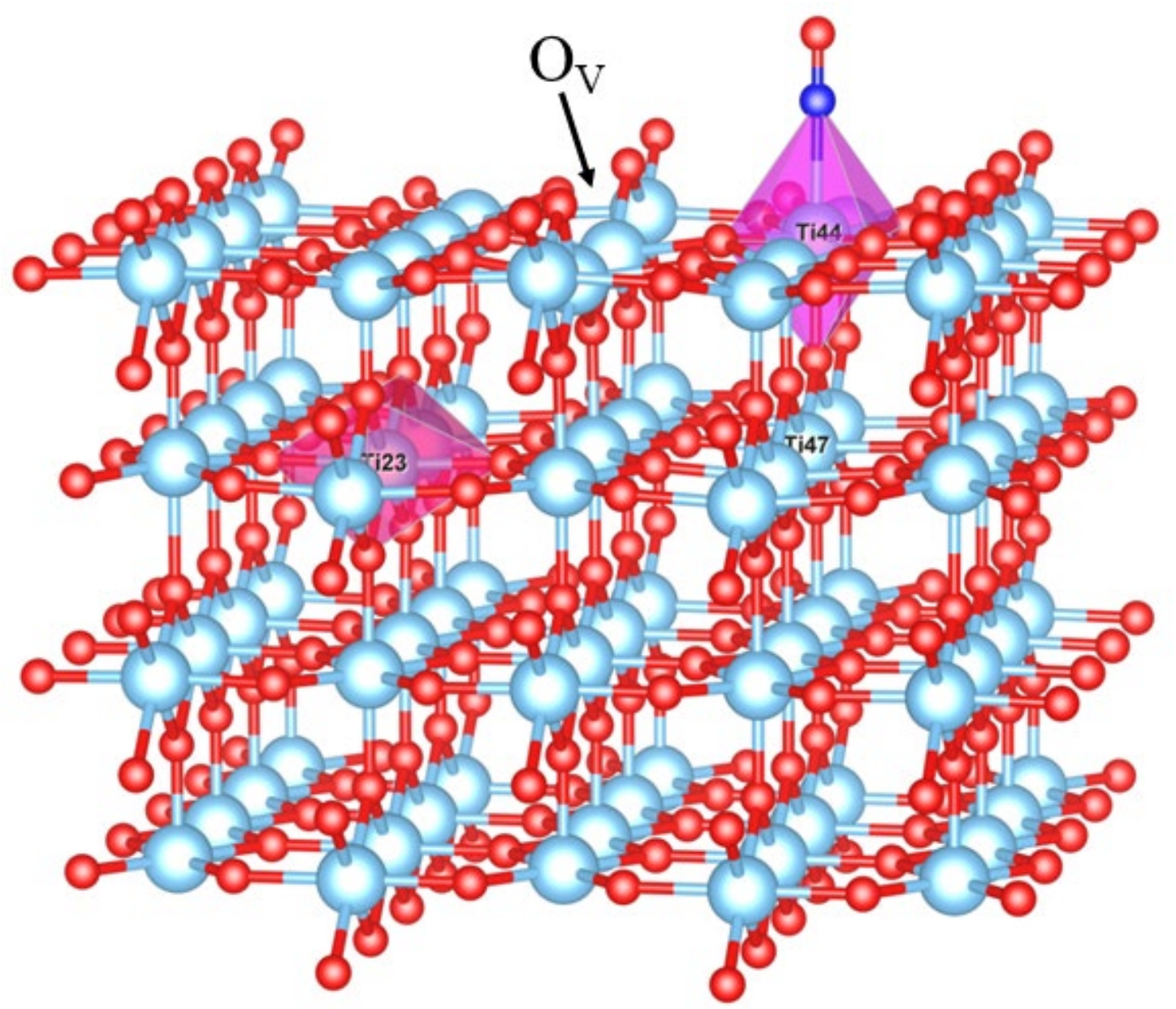

Figure S6. A CO molecule adsorbed on the reduced rutile $\mathrm{TiO}_{2}(110)$ surface pins one of the two polarons at the surface $\left(\mathrm{Ti}_{44}\right)$, while leaving the other polaron at the sublayer $\left(\mathrm{Ti}_{23}\right)$. Compare with Figure S1. 

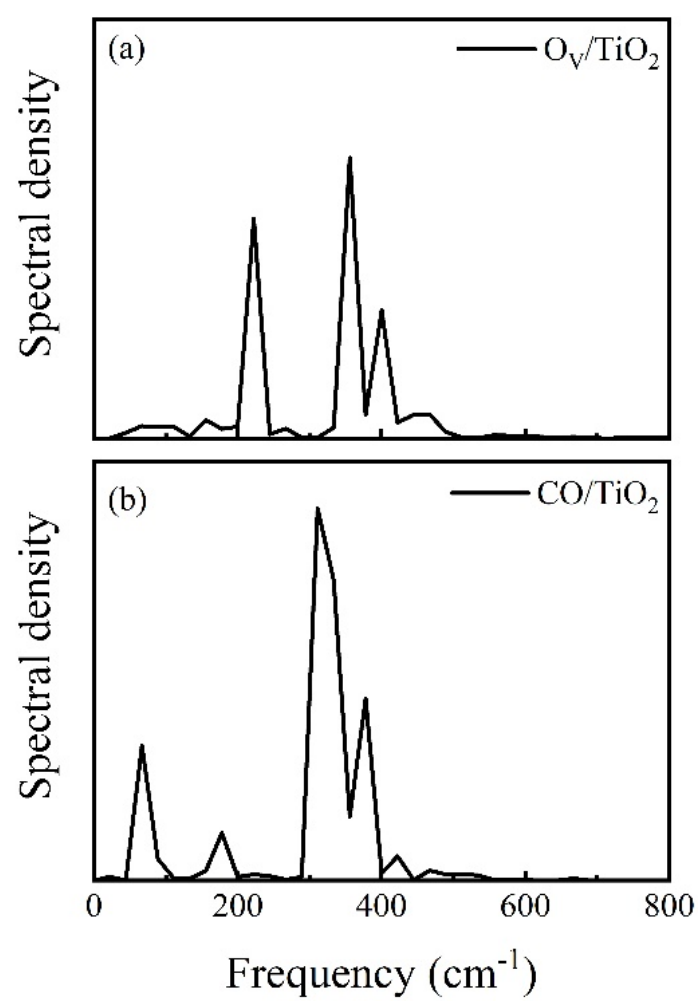

Figure S7. Spectral densities obtained by Fourier transforms of autocorrelation functions of fluctuations of the energy gaps between the VBM and trap1 in (a) $\mathrm{O}_{\mathrm{V}} / \mathrm{TiO}_{2}$ and (b) $\mathrm{CO} / \mathrm{TiO}_{2}$. 


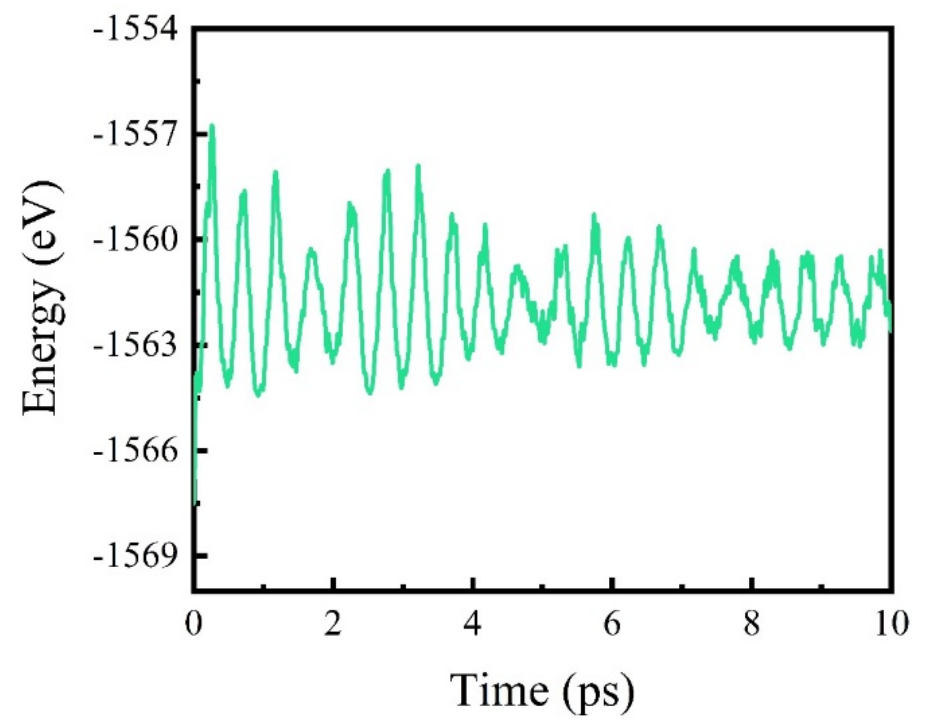

Figure S8. Evolution of the total energy during the 10 ps AIMD trajectory of the $\mathrm{CO} / \mathrm{TiO}_{2}$ system. 


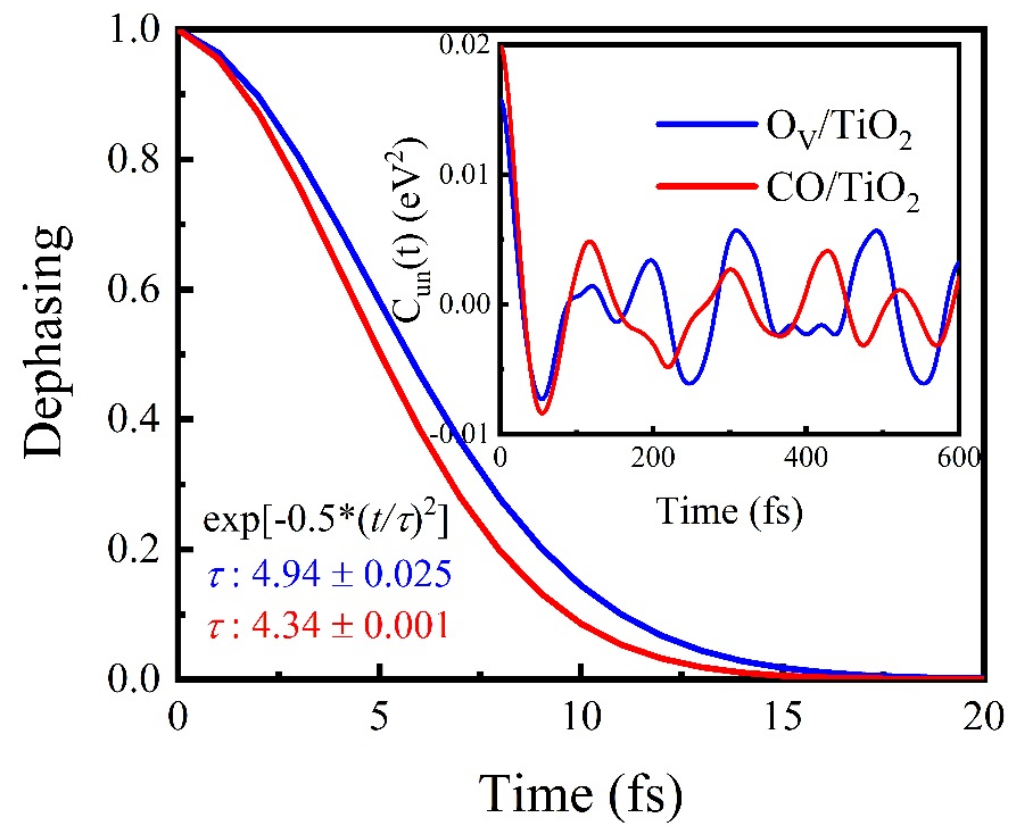

Figure S9. Pure-dephasing functions for transitions between the VBM and the trap1 state in the $\mathrm{O}_{\mathrm{V}} / \mathrm{TiO}_{2}$ and $\mathrm{CO} / \mathrm{TiO}_{2}$ systems. The inserts show the unnormalized autocorrelation functions, $\mathrm{C}_{\mathrm{un}}(\mathrm{t})$, of fluctuations of the energy gaps between the VBM and the trap1 state. The initial value, $C_{u n}(0)$, represents square of the gap fluctuation. Larger initial values lead to faster pure-dephasing. The standard deviations in the fitted timescales are labeled in the plot. 

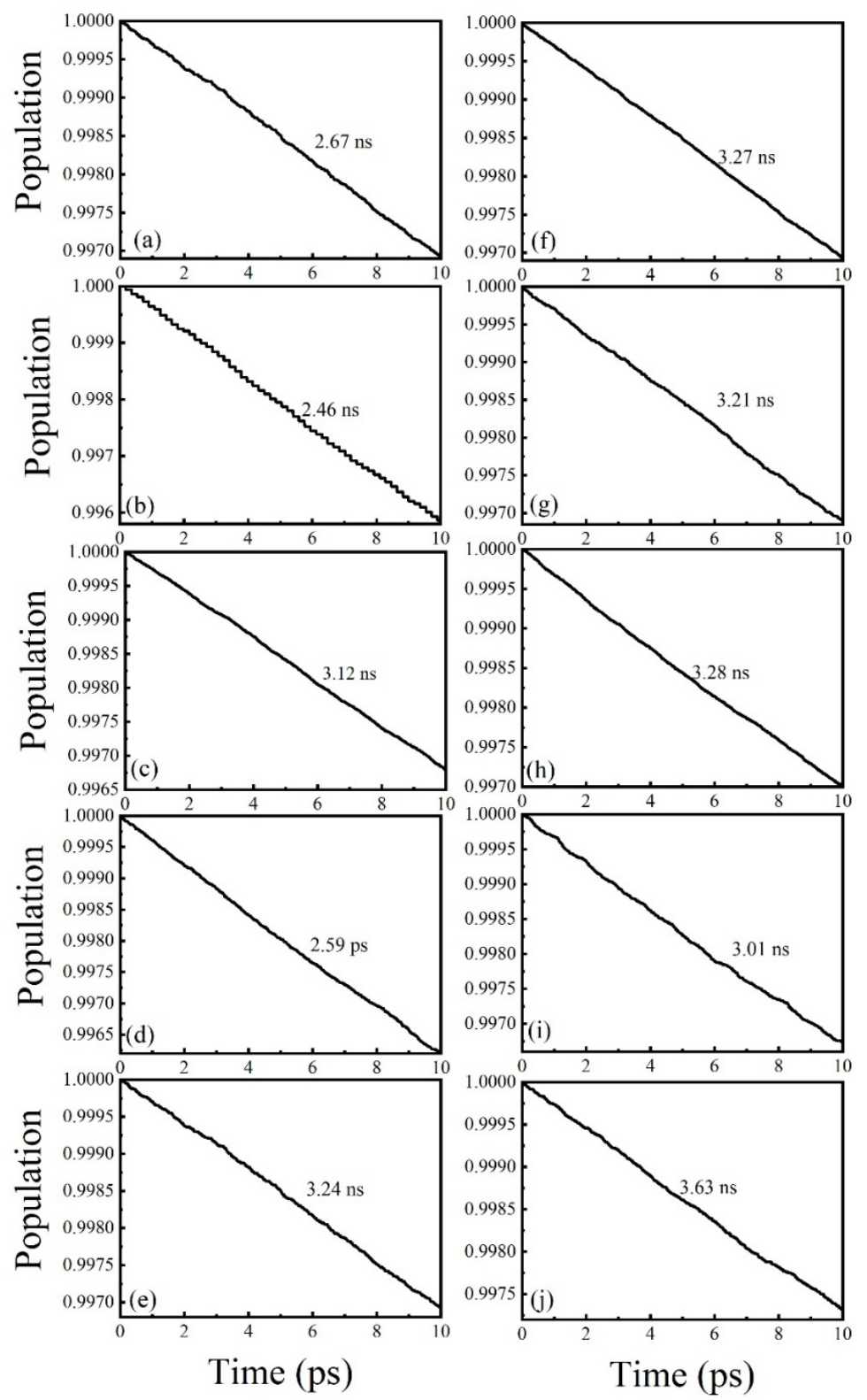

Figure S10. The electron-hole recombination dynamics in the (a-e) $\mathrm{O}_{\mathrm{V}} / \mathrm{TiO}_{2}$ and $(\mathbf{f}-\mathbf{j}) \mathrm{CO} / \mathrm{TiO}_{2}$ systems, obtained with 200 initial configurations by separating the 1000 initial configurations into 5 equal parts. The obtained time scales are similar with the result obtained using 1000 initial configurations, as shown in Figure in the main text. The standard deviations in the recombination times computed over the ensembles of these simulations are 0.31 and $0.20 \mathrm{~ns}$ for the $\mathrm{O}_{\mathrm{v}} / \mathrm{TiO}_{2}$ and $\mathrm{CO} / \mathrm{TiO}_{2}$ systems, respectively. 
Title

Ov two subsurface EPs

$\begin{array}{ll}\text { Lattice type } & \text { P } \\ \text { Space group name } & \text { P 1 } \\ \text { Space group number } & 1 \\ \text { Setting number } & 1\end{array}$

Lattice parameters

a $\quad b \quad$ c alpha beta gamma

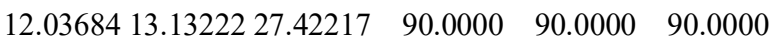

Unit-cell volume $=4334.634578 \AA \wedge 3$

Structure parameters

\begin{tabular}{|c|c|c|c|c|c|c|c|c|}
\hline & & $\mathrm{x}$ & $\mathrm{y}$ & $\mathrm{z}$ & Occ. & B & Site & Sym. \\
\hline $1 \mathrm{O}$ & $\mathrm{O} 1$ & 0.00495 & 0.25250 & 0.02237 & 1.000 & 1.000 & $1 \mathrm{a}$ & 1 \\
\hline $2 \mathrm{O}$ & $\mathrm{O} 2$ & 0.00494 & 0.00254 & 0.13923 & 1.000 & 1.000 & $1 \mathrm{a}$ & 1 \\
\hline $3 \mathrm{O}$ & $\mathrm{O} 3$ & 0.00551 & 0.25263 & 0.26107 & 1.000 & 1.000 & $1 \mathrm{a}$ & 1 \\
\hline $4 \mathrm{O}$ & $\mathrm{O} 4$ & 0.00492 & 0.00224 & 0.38239 & 1.000 & 1.000 & $1 \mathrm{a}$ & 1 \\
\hline $5 \mathrm{O}$ & O5 & 0.00495 & 0.25250 & 0.11620 & 1.000 & 1.000 & $1 \mathrm{a}$ & 1 \\
\hline 60 & O6 & 0.00495 & 0.00267 & 0.23453 & 1.000 & 1.000 & $1 \mathrm{a}$ & 1 \\
\hline $7 \mathrm{O}$ & $\mathrm{O} 7$ & 0.00619 & 0.25412 & 0.35527 & 1.000 & 1.000 & $1 \mathrm{a}$ & 1 \\
\hline 80 & O8 & 0.00484 & 0.00245 & 0.47600 & 1.000 & 1.000 & $1 \mathrm{a}$ & 1 \\
\hline 90 & O9 & 0.12994 & 0.10046 & 0.06928 & 1.000 & 1.000 & $1 \mathrm{a}$ & 1 \\
\hline $10 \mathrm{O}$ & $\mathrm{O} 10$ & 0.13000 & 0.35166 & 0.18821 & 1.000 & 1.000 & $1 \mathrm{a}$ & 1 \\
\hline $11 \mathrm{O}$ & O11 & 0.12978 & 0.10157 & 0.30879 & 1.000 & 1.000 & $1 \mathrm{a}$ & 1 \\
\hline $12 \mathrm{O}$ & $\mathrm{O} 12$ & 0.12961 & 0.34705 & 0.43551 & 1.000 & 1.000 & $1 \mathrm{a}$ & 1 \\
\hline $13 \mathrm{O}$ & $\mathrm{O} 13$ & 0.12994 & 0.40453 & 0.06928 & 1.000 & 1.000 & $1 \mathrm{a}$ & 1 \\
\hline $14 \mathrm{O}$ & $\mathrm{O} 14$ & 0.12998 & 0.15474 & 0.18840 & 1.000 & 1.000 & $1 \mathrm{a}$ & 1 \\
\hline $15 \mathrm{O}$ & O15 & 0.12915 & 0.40448 & 0.30816 & 1.000 & 1.000 & $1 \mathrm{a}$ & 1 \\
\hline $16 \mathrm{O}$ & O16 & 0.13063 & 0.15935 & 0.43405 & 1.000 & 1.000 & $1 \mathrm{a}$ & 1 \\
\hline $17 \mathrm{O}$ & $\mathrm{O} 17$ & 0.25495 & 0.25250 & 0.02237 & 1.000 & 1.000 & $1 \mathrm{a}$ & 1 \\
\hline $18 \mathrm{O}$ & O18 & 0.25489 & 0.00228 & 0.13927 & 1.000 & 1.000 & 1a & 1 \\
\hline $19 \mathrm{O}$ & O19 & 0.25409 & 0.25370 & 0.26114 & 1.000 & 1.000 & $1 \mathrm{a}$ & 1 \\
\hline $20 \mathrm{O}$ & $\mathrm{O} 20$ & 0.25505 & 0.00270 & 0.38250 & 1.000 & 1.000 & $1 \mathrm{a}$ & 1 \\
\hline $21 \mathrm{O}$ & $\mathrm{O} 21$ & 0.25495 & 0.25250 & 0.11620 & 1.000 & 1.000 & $1 \mathrm{a}$ & 1 \\
\hline $22 \mathrm{O}$ & $\mathrm{O} 22$ & 0.25470 & 0.00267 & 0.23445 & 1.000 & 1.000 & $1 \mathrm{a}$ & 1 \\
\hline $23 \mathrm{O}$ & $\mathrm{O} 23$ & 0.25340 & 0.25410 & 0.35553 & 1.000 & 1.000 & $1 \mathrm{a}$ & 1 \\
\hline $24 \mathrm{O}$ & $\mathrm{O} 24$ & 0.25496 & 0.00171 & 0.47611 & 1.000 & 1.000 & $1 \mathrm{a}$ & 1 \\
\hline $25 \mathrm{O}$ & $\mathrm{O} 25$ & 0.37995 & 0.10046 & 0.06928 & 1.000 & 1.000 & $1 \mathrm{a}$ & 1 \\
\hline $26 \mathrm{O}$ & $\mathrm{O} 26$ & 0.37904 & 0.35230 & 0.18766 & 1.000 & 1.000 & $1 \mathrm{a}$ & 1 \\
\hline $27 \mathrm{O}$ & $\mathrm{O} 27$ & 0.37974 & 0.10157 & 0.30886 & 1.000 & 1.000 & $1 \mathrm{a}$ & 1 \\
\hline
\end{tabular}




\begin{tabular}{|c|c|c|c|c|c|c|c|}
\hline $28 \mathrm{O}$ & $\mathrm{O} 28$ & 0.38115 & 0.35134 & 0.43465 & 1.000 & 1.000 & $1 \mathrm{a}$ \\
\hline $29 \mathrm{O}$ & O29 & 0.37995 & 0.40453 & 0.06928 & 1.000 & 1.000 & $1 \mathrm{a}$ \\
\hline $30 \mathrm{O}$ & $\mathrm{O} 30$ & 0.37907 & 0.15449 & 0.18796 & 1.000 & 1.000 & $1 \mathrm{a}$ \\
\hline $31 \mathrm{O}$ & $\mathrm{O} 31$ & 0.37946 & 0.40493 & 0.30962 & 1.000 & 1.000 & $1 \mathrm{a}$ \\
\hline $32 \mathrm{O}$ & $\mathrm{O} 32$ & 0.37909 & 0.16104 & 0.43525 & 1.000 & 1.000 & $1 \mathrm{a}$ \\
\hline $33 \mathrm{O}$ & $\mathrm{O} 33$ & 0.50494 & 0.25250 & 0.02237 & 1.000 & 1.000 & $1 \mathrm{a}$ \\
\hline $34 \mathrm{O}$ & $\mathrm{O} 34$ & 0.50495 & 0.00253 & 0.13933 & 1.000 & 1.000 & $1 \mathrm{a}$ \\
\hline $35 \mathrm{O}$ & $\mathrm{O} 35$ & 0.49870 & 0.25415 & 0.25932 & 1.000 & 1.000 & $1 \mathrm{a}$ \\
\hline 360 & $\mathrm{O} 36$ & 0.50492 & 0.00219 & 0.38263 & 1.000 & 1.000 & $1 \mathrm{a}$ \\
\hline $37 \mathrm{O}$ & $\mathrm{O} 37$ & 0.50494 & 0.25250 & 0.11620 & 1.000 & 1.000 & $1 \mathrm{a}$ \\
\hline $38 \mathrm{O}$ & $\mathrm{O} 38$ & 0.50494 & 0.00265 & 0.23444 & 1.000 & 1.000 & $1 \mathrm{a}$ \\
\hline $39 \mathrm{O}$ & O39 & 0.49849 & 0.25318 & 0.35626 & 1.000 & 1.000 & $1 \mathrm{a}$ \\
\hline $40 \mathrm{O}$ & $\mathrm{O} 40$ & 0.50483 & 0.00238 & 0.47611 & 1.000 & 1.000 & $1 \mathrm{a}$ \\
\hline $41 \mathrm{O}$ & $\mathrm{O} 41$ & 0.62994 & 0.10046 & 0.06928 & 1.000 & 1.000 & $1 \mathrm{a}$ \\
\hline $42 \mathrm{O}$ & $\mathrm{O} 42$ & 0.62991 & 0.35192 & 0.18842 & 1.000 & 1.000 & $1 \mathrm{a}$ \\
\hline $43 \mathrm{O}$ & $\mathrm{O} 43$ & 0.63021 & 0.09888 & 0.30903 & 1.000 & 1.000 & $1 \mathrm{a}$ \\
\hline $44 \mathrm{O}$ & $\mathrm{O} 44$ & 0.62799 & 0.35102 & 0.43406 & 1.000 & 1.000 & $1 \mathrm{a}$ \\
\hline $45 \mathrm{O}$ & $\mathrm{O} 45$ & 0.62994 & 0.40453 & 0.06928 & 1.000 & 1.000 & $1 \mathrm{a}$ \\
\hline $46 \mathrm{O}$ & $\mathrm{O} 46$ & 0.62988 & 0.15478 & 0.18868 & 1.000 & 1.000 & $1 \mathrm{a}$ \\
\hline $47 \mathrm{O}$ & $\mathrm{O} 47$ & 0.63032 & 0.40695 & 0.31002 & 1.000 & 1.000 & $1 \mathrm{a}$ \\
\hline $48 \mathrm{O}$ & $\mathrm{O} 48$ & 0.62985 & 0.16089 & 0.43425 & 1.000 & 1.000 & $1 \mathrm{a}$ \\
\hline $49 \mathrm{O}$ & O49 & 0.75494 & 0.25250 & 0.02237 & 1.000 & 1.000 & $1 \mathrm{a}$ \\
\hline $50 \mathrm{O}$ & O50 & 0.75495 & 0.00272 & 0.13927 & 1.000 & 1.000 & $1 \mathrm{a}$ \\
\hline $51 \mathrm{O}$ & O51 & 0.76117 & 0.25372 & 0.25935 & 1.000 & 1.000 & $1 \mathrm{a}$ \\
\hline $52 \mathrm{O}$ & $\mathrm{O} 52$ & 0.75471 & 0.00191 & 0.38250 & 1.000 & 1.000 & $1 \mathrm{a}$ \\
\hline $53 \mathrm{O}$ & O53 & 0.75494 & 0.25250 & 0.11620 & 1.000 & 1.000 & $1 \mathrm{a}$ \\
\hline $54 \mathrm{O}$ & O54 & 0.75521 & 0.00261 & 0.23446 & 1.000 & 1.000 & $1 \mathrm{a}$ \\
\hline $55 \mathrm{O}$ & O55 & 0.76139 & 0.25427 & 0.35664 & 1.000 & 1.000 & $1 \mathrm{a}$ \\
\hline $56 \mathrm{O}$ & O56 & 0.75454 & 0.00299 & 0.47612 & 1.000 & 1.000 & $1 \mathrm{a}$ \\
\hline $57 \mathrm{O}$ & O57 & 0.87994 & 0.10046 & 0.06928 & 1.000 & 1.000 & $1 \mathrm{a}$ \\
\hline $58 \mathrm{O}$ & O58 & 0.88073 & 0.35189 & 0.18769 & 1.000 & 1.000 & $1 \mathrm{a}$ \\
\hline $59 \mathrm{O}$ & O59 & 0.88019 & 0.10084 & 0.30897 & 1.000 & 1.000 & $1 \mathrm{a}$ \\
\hline $60 \mathrm{O}$ & O60 & 0.88122 & 0.34691 & 0.43586 & 1.000 & 1.000 & $1 \mathrm{a}$ \\
\hline $61 \mathrm{O}$ & O61 & 0.87994 & 0.40453 & 0.06928 & 1.000 & 1.000 & $1 \mathrm{a}$ \\
\hline $62 \mathrm{O}$ & O62 & 0.88075 & 0.15443 & 0.18788 & 1.000 & 1.000 & $1 \mathrm{a}$ \\
\hline $63 \mathrm{O}$ & O63 & 0.88093 & 0.40511 & 0.30821 & 1.000 & 1.000 & $1 \mathrm{a}$ \\
\hline $64 \mathrm{O}$ & O64 & 0.88018 & 0.15925 & 0.43431 & 1.000 & 1.000 & $1 \mathrm{a}$ \\
\hline $65 \mathrm{O}$ & O65 & 0.00495 & 0.75250 & 0.02237 & 1.000 & 1.000 & $1 \mathrm{a}$ \\
\hline $66 \mathrm{O}$ & O66 & 0.00497 & 0.50250 & 0.13927 & 1.000 & 1.000 & $1 \mathrm{a}$ \\
\hline $67 \mathrm{O}$ & O67 & 0.00437 & 0.75223 & 0.26106 & 1.000 & 1.000 & $1 \mathrm{a}$ \\
\hline $68 \mathrm{O}$ & O68 & 0.00496 & 0.50237 & 0.38492 & 1.000 & 1.000 & $1 \mathrm{a}$ \\
\hline $69 \mathrm{O}$ & O69 & 0.00495 & 0.75250 & 0.11620 & 1.000 & 1.000 & $1 \mathrm{a}$ \\
\hline $70 \mathrm{O}$ & $\mathrm{O} 70$ & 0.00495 & 0.50263 & 0.23424 & 1.000 & 1.000 & $1 \mathrm{a}$ \\
\hline $71 \mathrm{O}$ & O71 & 0.00380 & 0.75095 & 0.35525 & 1.000 & 1.000 & $1 \mathrm{a}$ \\
\hline
\end{tabular}




\begin{tabular}{|c|c|c|c|c|c|c|c|}
\hline $72 \mathrm{O}$ & $\mathrm{O} 72$ & 0.00498 & 0.50242 & 0.48015 & 1.000 & 1.000 & $1 \mathrm{a}$ \\
\hline $73 \mathrm{O}$ & $\mathrm{O} 73$ & 0.12994 & 0.60046 & 0.06928 & 1.000 & 1.000 & $1 \mathrm{a}$ \\
\hline $74 \mathrm{O}$ & O74 & 0.12910 & 0.85085 & 0.18795 & 1.000 & 1.000 & $1 \mathrm{a}$ \\
\hline $75 \mathrm{O}$ & $\mathrm{O} 75$ & 0.12906 & 0.59995 & 0.30834 & 1.000 & 1.000 & $1 \mathrm{a}$ \\
\hline $76 \mathrm{O}$ & O76 & 0.12966 & 0.84547 & 0.43442 & 1.000 & 1.000 & $1 \mathrm{a}$ \\
\hline $77 \mathrm{O}$ & O77 & 0.12994 & 0.90453 & 0.06928 & 1.000 & 1.000 & $1 \mathrm{a}$ \\
\hline $78 \mathrm{O}$ & $\mathrm{O} 78$ & 0.12926 & 0.65336 & 0.18766 & 1.000 & 1.000 & 1a \\
\hline $79 \mathrm{O}$ & O79 & 0.12980 & 0.90425 & 0.30882 & 1.000 & 1.000 & $1 \mathrm{a}$ \\
\hline $80 \mathrm{O}$ & O80 & 0.12883 & 0.65780 & 0.43580 & 1.000 & 1.000 & $1 \mathrm{a}$ \\
\hline $81 \mathrm{O}$ & O81 & 0.25495 & 0.75250 & 0.02237 & 1.000 & 1.000 & $1 \mathrm{a}$ \\
\hline $82 \mathrm{O}$ & O82 & 0.25497 & 0.50274 & 0.13925 & 1.000 & 1.000 & $1 \mathrm{a}$ \\
\hline $83 \mathrm{O}$ & O83 & 0.24884 & 0.75106 & 0.25932 & 1.000 & 1.000 & $1 \mathrm{a}$ \\
\hline $84 \mathrm{O}$ & O84 & 0.24497 & 0.50208 & 0.38053 & 1.000 & 1.000 & $1 \mathrm{a}$ \\
\hline $85 \mathrm{O}$ & O85 & 0.25495 & 0.75250 & 0.11620 & 1.000 & 1.000 & $1 \mathrm{a}$ \\
\hline $86 \mathrm{O}$ & O86 & 0.25507 & 0.50271 & 0.23430 & 1.000 & 1.000 & $1 \mathrm{a}$ \\
\hline $87 \mathrm{O}$ & O87 & 0.24848 & 0.75084 & 0.35664 & 1.000 & 1.000 & $1 \mathrm{a}$ \\
\hline $88 \mathrm{O}$ & O88 & 0.26017 & 0.50299 & 0.47380 & 1.000 & 1.000 & $1 \mathrm{a}$ \\
\hline $89 \mathrm{O}$ & O89 & 0.37995 & 0.60046 & 0.06928 & 1.000 & 1.000 & $1 \mathrm{a}$ \\
\hline $90 \mathrm{O}$ & O90 & 0.38011 & 0.85049 & 0.18871 & 1.000 & 1.000 & 1a \\
\hline $91 \mathrm{O}$ & O91 & 0.37969 & 0.59796 & 0.31011 & 1.000 & 1.000 & 1a \\
\hline $92 \mathrm{O}$ & O92 & 0.38009 & 0.84382 & 0.43433 & 1.000 & 1.000 & 1a \\
\hline $93 \mathrm{O}$ & O93 & 0.37995 & 0.90453 & 0.06928 & 1.000 & 1.000 & $1 \mathrm{a}$ \\
\hline $94 \mathrm{O}$ & O94 & 0.37995 & 0.65335 & 0.18831 & 1.000 & 1.000 & $1 \mathrm{a}$ \\
\hline $95 \mathrm{O}$ & O95 & 0.37978 & 0.90606 & 0.30888 & 1.000 & 1.000 & 1a \\
\hline 960 & O96 & 0.38191 & 0.65367 & 0.43380 & 1.000 & 1.000 & 1a \\
\hline $97 \mathrm{O}$ & O97 & 0.50494 & 0.75250 & 0.02237 & 1.000 & 1.000 & 1a \\
\hline $98 \mathrm{O}$ & O98 & 0.50498 & 0.50255 & 0.13953 & 1.000 & 1.000 & 1a \\
\hline $99 \mathrm{O}$ & O99 & 0.51114 & 0.75050 & 0.25931 & 1.000 & 1.000 & $1 \mathrm{a}$ \\
\hline $100 \mathrm{O}$ & O100 & 0.50497 & 0.50213 & 0.38652 & 1.000 & 1.000 & $1 \mathrm{a}$ \\
\hline $101 \mathrm{O}$ & O101 & 0.50494 & 0.75250 & 0.11620 & 1.000 & 1.000 & $1 \mathrm{a}$ \\
\hline $102 \mathrm{O}$ & O102 & 0.50494 & 0.50263 & 0.23508 & 1.000 & 1.000 & 1a \\
\hline $103 \mathrm{O}$ & O103 & 0.51145 & 0.75192 & 0.35623 & 1.000 & 1.000 & $1 \mathrm{a}$ \\
\hline $104 \mathrm{O}$ & O104 & 0.62994 & 0.60046 & 0.06928 & 1.000 & 1.000 & 1a \\
\hline $105 \mathrm{O}$ & O105 & 0.63083 & 0.85079 & 0.18806 & 1.000 & 1.000 & 1a \\
\hline $106 \mathrm{O}$ & O106 & 0.63040 & 0.59998 & 0.30975 & 1.000 & 1.000 & $1 \mathrm{a}$ \\
\hline $107 \mathrm{O}$ & O107 & 0.63075 & 0.84367 & 0.43533 & 1.000 & 1.000 & $1 \mathrm{a}$ \\
\hline $108 \mathrm{O}$ & O108 & 0.62994 & 0.90453 & 0.06928 & 1.000 & 1.000 & 1a \\
\hline $109 \mathrm{O}$ & O109 & 0.63099 & 0.65300 & 0.18764 & 1.000 & 1.000 & 1a \\
\hline $110 \mathrm{O}$ & O110 & 0.63031 & 0.90335 & 0.30870 & 1.000 & 1.000 & 1a \\
\hline $111 \mathrm{O}$ & O111 & 0.62873 & 0.65337 & 0.43453 & 1.000 & 1.000 & 1a \\
\hline $112 \mathrm{O}$ & O112 & 0.75494 & 0.75250 & 0.02237 & 1.000 & 1.000 & 1a \\
\hline $113 \mathrm{O}$ & O113 & 0.75488 & 0.50225 & 0.13926 & 1.000 & 1.000 & $1 \mathrm{a}$ \\
\hline $114 \mathrm{O}$ & O114 & 0.75586 & 0.75109 & 0.26112 & 1.000 & 1.000 & 1a \\
\hline $115 \mathrm{O}$ & O115 & 0.76488 & 0.50227 & 0.38056 & 1.000 & 1.000 & 1a \\
\hline
\end{tabular}




\begin{tabular}{|c|c|c|c|c|c|c|c|}
\hline $116 \mathrm{O}$ & O116 & 0.75494 & 0.75250 & 0.11620 & 1.000 & 1.000 & $1 \mathrm{a}$ \\
\hline $117 \mathrm{O}$ & O117 & 0.75487 & 0.50250 & 0.23431 & 1.000 & 1.000 & 1a \\
\hline $118 \mathrm{O}$ & O118 & 0.75648 & 0.75096 & 0.35550 & 1.000 & 1.000 & 1a \\
\hline $119 \mathrm{O}$ & O119 & 0.74975 & 0.50185 & 0.47383 & 1.000 & 1.000 & 1a \\
\hline $120 \mathrm{O}$ & $\mathrm{O} 120$ & 0.87994 & 0.60046 & 0.06928 & 1.000 & 1.000 & $1 \mathrm{a}$ \\
\hline $121 \mathrm{O}$ & O121 & 0.88001 & 0.85052 & 0.18844 & 1.000 & 1.000 & 1a \\
\hline $122 \mathrm{O}$ & $\mathrm{O} 122$ & 0.88074 & 0.60060 & 0.30832 & 1.000 & 1.000 & $1 \mathrm{a}$ \\
\hline $123 \mathrm{O}$ & $\mathrm{O} 123$ & 0.87933 & 0.84538 & 0.43410 & 1.000 & 1.000 & 1a \\
\hline $124 \mathrm{O}$ & $\mathrm{O} 124$ & 0.87994 & 0.90453 & 0.06928 & 1.000 & 1.000 & 1a \\
\hline $125 \mathrm{O}$ & $\mathrm{O} 125$ & 0.87987 & 0.65357 & 0.18814 & 1.000 & 1.000 & 1a \\
\hline $126 \mathrm{O}$ & O126 & 0.88021 & 0.90347 & 0.30869 & 1.000 & 1.000 & $1 \mathrm{a}$ \\
\hline $127 \mathrm{O}$ & $\mathrm{O} 127$ & 0.88028 & 0.65768 & 0.43539 & 1.000 & 1.000 & $1 \mathrm{a}$ \\
\hline $128 \mathrm{Ti}$ & Ti1 & 0.00495 & 0.00250 & 0.06928 & 1.000 & 1.000 & 1a \\
\hline $129 \mathrm{Ti}$ & Ti2 & 0.00499 & 0.25232 & 0.18538 & 1.000 & 1.000 & 1a \\
\hline $130 \mathrm{Ti}$ & Ti3 & 0.00505 & 0.00260 & 0.31324 & 1.000 & 1.000 & $1 \mathrm{a}$ \\
\hline $131 \mathrm{Ti}$ & Ti4 & 0.00533 & 0.25242 & 0.42222 & 1.000 & 1.000 & 1a \\
\hline $132 \mathrm{Ti}$ & Ti5 & 0.12994 & 0.25250 & 0.06928 & 1.000 & 1.000 & 1a \\
\hline $133 \mathrm{Ti}$ & Ti6 & 0.12985 & 0.00249 & 0.18978 & 1.000 & 1.000 & 1a \\
\hline $134 \mathrm{Ti}$ & Ti7 & 0.12963 & 0.25159 & 0.30468 & 1.000 & 1.000 & 1a \\
\hline $135 \mathrm{Ti}$ & Ti8 & 0.12982 & 0.00233 & 0.43587 & 1.000 & 1.000 & $1 \mathrm{a}$ \\
\hline $136 \mathrm{Ti}$ & Ti9 & 0.25495 & 0.00250 & 0.06928 & 1.000 & 1.000 & $1 \mathrm{a}$ \\
\hline $137 \mathrm{Ti}$ & Ti10 & 0.25480 & 0.25284 & 0.18534 & 1.000 & 1.000 & 1a \\
\hline $138 \mathrm{Ti}$ & Til1 & 0.25566 & 0.00245 & 0.31317 & 1.000 & 1.000 & 1a \\
\hline $139 \mathrm{Ti}$ & Ti12 & 0.25439 & 0.25306 & 0.42243 & 1.000 & 1.000 & 1a \\
\hline $140 \mathrm{Ti}$ & Ti13 & 0.37995 & 0.25250 & 0.06928 & 1.000 & 1.000 & $1 \mathrm{a}$ \\
\hline $141 \mathrm{Ti}$ & Ti14 & 0.37995 & 0.00296 & 0.18985 & 1.000 & 1.000 & $1 \mathrm{a}$ \\
\hline $142 \mathrm{Ti}$ & Ti15 & 0.38201 & 0.25138 & 0.30386 & 1.000 & 1.000 & $1 \mathrm{a}$ \\
\hline $143 \mathrm{Ti}$ & Ti16 & 0.37970 & 0.00239 & 0.43604 & 1.000 & 1.000 & 1a \\
\hline $144 \mathrm{Ti}$ & Ti17 & 0.50494 & 0.00250 & 0.06928 & 1.000 & 1.000 & 1a \\
\hline $145 \mathrm{Ti}$ & Ti18 & 0.50500 & 0.25242 & 0.18600 & 1.000 & 1.000 & 1a \\
\hline $146 \mathrm{Ti}$ & Ti19 & 0.50510 & 0.00240 & 0.31322 & 1.000 & 1.000 & 1a \\
\hline $147 \mathrm{Ti}$ & Ti20 & 0.50411 & 0.25348 & 0.42251 & 1.000 & 1.000 & $1 \mathrm{a}$ \\
\hline $148 \mathrm{Ti}$ & Ti21 & 0.62994 & 0.25250 & 0.06928 & 1.000 & 1.000 & $1 \mathrm{a}$ \\
\hline $149 \mathrm{Ti}$ & Ti22 & 0.62997 & 0.00259 & 0.18984 & 1.000 & 1.000 & 1a \\
\hline $150 \mathrm{Ti}$ & Ti23 & 0.63002 & 0.25170 & 0.30666 & 1.000 & 1.000 & 1a \\
\hline $151 \mathrm{Ti}$ & Ti24 & 0.62974 & 0.00250 & 0.43593 & 1.000 & 1.000 & 1a \\
\hline $152 \mathrm{Ti}$ & Ti25 & 0.75494 & 0.00250 & 0.06928 & 1.000 & 1.000 & $1 \mathrm{a}$ \\
\hline $153 \mathrm{Ti}$ & Ti26 & 0.75491 & 0.25273 & 0.18599 & 1.000 & 1.000 & $1 \mathrm{a}$ \\
\hline $154 \mathrm{Ti}$ & Ti27 & 0.75448 & 0.00269 & 0.31318 & 1.000 & 1.000 & $1 \mathrm{a}$ \\
\hline $155 \mathrm{Ti}$ & Ti28 & 0.75601 & 0.25275 & 0.42304 & 1.000 & 1.000 & 1a \\
\hline $156 \mathrm{Ti}$ & Ti29 & 0.87994 & 0.25250 & 0.06928 & 1.000 & 1.000 & 1a \\
\hline $157 \mathrm{Ti}$ & Ti30 & 0.88006 & 0.00296 & 0.18980 & 1.000 & 1.000 & 1a \\
\hline $158 \mathrm{Ti}$ & Ti31 & 0.87778 & 0.25173 & 0.30393 & 1.000 & 1.000 & $1 \mathrm{a}$ \\
\hline $159 \mathrm{Ti}$ & Ti32 & 0.87962 & 0.00252 & 0.43599 & 1.000 & 1.000 & 1a \\
\hline
\end{tabular}




\begin{tabular}{|c|c|c|c|c|c|c|c|}
\hline $160 \mathrm{Ti}$ & Ti33 & 0.00495 & 0.50250 & 0.06928 & 1.000 & 1.000 & $1 \mathrm{a}$ \\
\hline $161 \mathrm{Ti}$ & Ti34 & 0.00497 & 0.75265 & 0.18539 & 1.000 & 1.000 & $1 \mathrm{a}$ \\
\hline $162 \mathrm{Ti}$ & Ti35 & 0.00503 & 0.50251 & 0.31461 & 1.000 & 1.000 & 1a \\
\hline $163 \mathrm{Ti}$ & Ti36 & 0.00470 & 0.75232 & 0.42218 & 1.000 & 1.000 & $1 \mathrm{a}$ \\
\hline $164 \mathrm{Ti}$ & Ti37 & 0.12994 & 0.75250 & 0.06928 & 1.000 & 1.000 & $1 \mathrm{a}$ \\
\hline $165 \mathrm{Ti}$ & Ti38 & 0.12801 & 0.50298 & 0.18901 & 1.000 & 1.000 & 1a \\
\hline $166 \mathrm{Ti}$ & Ti39 & 0.13219 & 0.75364 & 0.30387 & 1.000 & 1.000 & $1 \mathrm{a}$ \\
\hline $167 \mathrm{Ti}$ & Ti40 & 0.12603 & 0.50265 & 0.43841 & 1.000 & 1.000 & $1 \mathrm{a}$ \\
\hline $168 \mathrm{Ti}$ & Ti41 & 0.25495 & 0.50250 & 0.06928 & 1.000 & 1.000 & $1 \mathrm{a}$ \\
\hline $169 \mathrm{Ti}$ & Ti42 & 0.25498 & 0.75303 & 0.18597 & 1.000 & 1.000 & $1 \mathrm{a}$ \\
\hline $170 \mathrm{Ti}$ & Ti43 & 0.25359 & 0.50265 & 0.30640 & 1.000 & 1.000 & 1a \\
\hline $171 \mathrm{Ti}$ & Ti44 & 0.25391 & 0.75220 & 0.42303 & 1.000 & 1.000 & 1a \\
\hline $172 \mathrm{Ti}$ & Ti45 & 0.37995 & 0.75250 & 0.06928 & 1.000 & 1.000 & 1a \\
\hline $173 \mathrm{Ti}$ & Ti46 & 0.38040 & 0.50271 & 0.18830 & 1.000 & 1.000 & 1a \\
\hline $174 \mathrm{Ti}$ & Ti47 & 0.37989 & 0.75328 & 0.30668 & 1.000 & 1.000 & $1 \mathrm{a}$ \\
\hline $175 \mathrm{Ti}$ & Ti48 & 0.36153 & 0.50259 & 0.42311 & 1.000 & 1.000 & $1 \mathrm{a}$ \\
\hline $176 \mathrm{Ti}$ & Ti49 & 0.50494 & 0.50250 & 0.06928 & 1.000 & 1.000 & $1 \mathrm{a}$ \\
\hline $177 \mathrm{Ti}$ & Ti50 & 0.50502 & 0.75282 & 0.18599 & 1.000 & 1.000 & 1a \\
\hline $178 \mathrm{Ti}$ & Ti51 & 0.50500 & 0.50229 & 0.31037 & 1.000 & 1.000 & $1 \mathrm{a}$ \\
\hline $179 \mathrm{Ti}$ & Ti52 & 0.50578 & 0.75132 & 0.42246 & 1.000 & 1.000 & $1 \mathrm{a}$ \\
\hline $180 \mathrm{Ti}$ & Ti53 & 0.62994 & 0.75250 & 0.06928 & 1.000 & 1.000 & $1 \mathrm{a}$ \\
\hline $181 \mathrm{Ti}$ & Ti54 & 0.62957 & 0.50288 & 0.18833 & 1.000 & 1.000 & $1 \mathrm{a}$ \\
\hline $182 \mathrm{Ti}$ & Ti55 & 0.62793 & 0.75349 & 0.30379 & 1.000 & 1.000 & 1a \\
\hline $183 \mathrm{Ti}$ & Ti56 & 0.64831 & 0.50220 & 0.42313 & 1.000 & 1.000 & 1a \\
\hline $184 \mathrm{Ti}$ & Ti57 & 0.75494 & 0.50250 & 0.06928 & 1.000 & 1.000 & 1a \\
\hline $185 \mathrm{Ti}$ & Ti58 & 0.75520 & 0.75284 & 0.18534 & 1.000 & 1.000 & 1a \\
\hline $186 \mathrm{Ti}$ & Ti59 & 0.75641 & 0.50266 & 0.30643 & 1.000 & 1.000 & $1 \mathrm{a}$ \\
\hline $187 \mathrm{Ti}$ & Ti60 & 0.75556 & 0.75199 & 0.42239 & 1.000 & 1.000 & 1a \\
\hline $188 \mathrm{Ti}$ & Ti61 & 0.87994 & 0.75250 & 0.06928 & 1.000 & 1.000 & 1a \\
\hline $189 \mathrm{Ti}$ & Ti62 & 0.88201 & 0.50232 & 0.18898 & 1.000 & 1.000 & $1 \mathrm{a}$ \\
\hline $190 \mathrm{Ti}$ & Ti63 & 0.88026 & 0.75364 & 0.30470 & 1.000 & 1.000 & 1a \\
\hline $191 \mathrm{Ti}$ & Ti64 & 0.88385 & 0.50223 & 0.43848 & 1.000 & 1.000 & $1 \mathrm{a}$ \\
\hline
\end{tabular}


Title CO@NNTi5c two subsurface EPs

$\begin{array}{ll}\text { Lattice type } & \text { P } \\ \text { Space group name } & \text { P 1 } \\ \text { Space group number } & 1 \\ \text { Setting number } & 1\end{array}$

Lattice parameters

a $\quad b \quad$ c alpha beta gamma

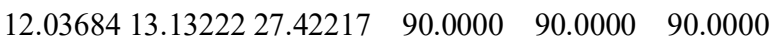

Unit-cell volume $=4334.634578 \AA \wedge 3$

Structure parameters

\begin{tabular}{|c|c|c|c|c|c|c|c|c|}
\hline & & $\mathrm{x}$ & $\mathrm{y}$ & z & Occ. & B & Site & Sym. \\
\hline $1 \mathrm{C}$ & $\mathrm{C} 1$ & 0.25297 & 0.75276 & 0.51373 & 1.000 & 1.000 & $1 \mathrm{a}$ & 1 \\
\hline $2 \mathrm{O}$ & $\mathrm{O} 1$ & 0.00495 & 0.25250 & 0.02237 & 1.000 & 1.000 & $1 \mathrm{a}$ & 1 \\
\hline 30 & $\mathrm{O} 2$ & 0.00498 & 0.00259 & 0.13922 & 1.000 & 1.000 & $1 \mathrm{a}$ & 1 \\
\hline $4 \mathrm{O}$ & $\mathrm{O} 3$ & 0.00550 & 0.25269 & 0.26110 & 1.000 & 1.000 & $1 \mathrm{a}$ & 1 \\
\hline $5 \mathrm{O}$ & $\mathrm{O} 4$ & 0.00477 & 0.00283 & 0.38234 & 1.000 & 1.000 & $1 \mathrm{a}$ & 1 \\
\hline 60 & O5 & 0.00495 & 0.25250 & 0.11620 & 1.000 & 1.000 & $1 \mathrm{a}$ & 1 \\
\hline $7 \mathrm{O}$ & O6 & 0.00504 & 0.00263 & 0.23450 & 1.000 & 1.000 & $1 \mathrm{a}$ & 1 \\
\hline $8 \mathrm{O}$ & $\mathrm{O} 7$ & 0.00623 & 0.25401 & 0.35539 & 1.000 & 1.000 & $1 \mathrm{a}$ & 1 \\
\hline 90 & O8 & 0.00492 & 0.00222 & 0.47600 & 1.000 & 1.000 & $1 \mathrm{a}$ & 1 \\
\hline $10 \mathrm{O}$ & O9 & 0.12994 & 0.10046 & 0.06928 & 1.000 & 1.000 & $1 \mathrm{a}$ & 1 \\
\hline $11 \mathrm{O}$ & $\mathrm{O} 10$ & 0.13005 & 0.35166 & 0.18820 & 1.000 & 1.000 & $1 \mathrm{a}$ & 1 \\
\hline $12 \mathrm{O}$ & O11 & 0.12978 & 0.10171 & 0.30865 & 1.000 & 1.000 & $1 \mathrm{a}$ & 1 \\
\hline $13 \mathrm{O}$ & $\mathrm{O} 12$ & 0.12959 & 0.34702 & 0.43574 & 1.000 & 1.000 & $1 \mathrm{a}$ & 1 \\
\hline $14 \mathrm{O}$ & $\mathrm{O} 13$ & 0.12994 & 0.40453 & 0.06928 & 1.000 & 1.000 & $1 \mathrm{a}$ & 1 \\
\hline $15 \mathrm{O}$ & $\mathrm{O} 14$ & 0.13004 & 0.15474 & 0.18841 & 1.000 & 1.000 & $1 \mathrm{a}$ & 1 \\
\hline $16 \mathrm{O}$ & O15 & 0.12918 & 0.40437 & 0.30813 & 1.000 & 1.000 & $1 \mathrm{a}$ & 1 \\
\hline $17 \mathrm{O}$ & O16 & 0.13073 & 0.15938 & 0.43440 & 1.000 & 1.000 & $1 \mathrm{a}$ & 1 \\
\hline $18 \mathrm{O}$ & $\mathrm{O} 17$ & 0.25495 & 0.25250 & 0.02237 & 1.000 & 1.000 & $1 \mathrm{a}$ & 1 \\
\hline $19 \mathrm{O}$ & O18 & 0.25495 & 0.00239 & 0.13926 & 1.000 & 1.000 & $1 \mathrm{a}$ & 1 \\
\hline $20 \mathrm{O}$ & O19 & 0.25414 & 0.25381 & 0.26117 & 1.000 & 1.000 & $1 \mathrm{a}$ & 1 \\
\hline $21 \mathrm{O}$ & $\mathrm{O} 20$ & 0.25505 & 0.00383 & 0.38245 & 1.000 & 1.000 & $1 \mathrm{a}$ & 1 \\
\hline $22 \mathrm{O}$ & $\mathrm{O} 21$ & 0.25495 & 0.25250 & 0.11620 & 1.000 & 1.000 & $1 \mathrm{a}$ & 1 \\
\hline $23 \mathrm{O}$ & $\mathrm{O} 22$ & 0.25474 & 0.00264 & 0.23443 & 1.000 & 1.000 & $1 \mathrm{a}$ & 1 \\
\hline $24 \mathrm{O}$ & $\mathrm{O} 23$ & 0.25344 & 0.25400 & 0.35565 & 1.000 & 1.000 & $1 \mathrm{a}$ & 1 \\
\hline $25 \mathrm{O}$ & $\mathrm{O} 24$ & 0.25494 & -0.00082 & 0.47600 & 1.000 & 1.000 & 1a & 1 \\
\hline $26 \mathrm{O}$ & $\mathrm{O} 25$ & 0.37995 & 0.10046 & 0.06928 & 1.000 & 1.000 & $1 \mathrm{a}$ & 1 \\
\hline $27 \mathrm{O}$ & $\mathrm{O} 26$ & 0.37909 & 0.35228 & 0.18767 & 1.000 & 1.000 & $1 \mathrm{a}$ & 1 \\
\hline
\end{tabular}




\begin{tabular}{|c|c|c|c|c|c|c|c|}
\hline $28 \mathrm{O}$ & $\mathrm{O} 27$ & 0.37985 & 0.10167 & 0.30865 & 1.000 & 1.000 & $1 \mathrm{a}$ \\
\hline $29 \mathrm{O}$ & $\mathrm{O} 28$ & 0.38106 & 0.35156 & 0.43495 & 1.000 & 1.000 & $1 \mathrm{a}$ \\
\hline $30 \mathrm{O}$ & $\mathrm{O} 29$ & 0.37995 & 0.40453 & 0.06928 & 1.000 & 1.000 & $1 \mathrm{a}$ \\
\hline $31 \mathrm{O}$ & $\mathrm{O} 30$ & 0.37916 & 0.15453 & 0.18803 & 1.000 & 1.000 & 1a \\
\hline $32 \mathrm{O}$ & $\mathrm{O} 31$ & 0.37956 & 0.40486 & 0.30965 & 1.000 & 1.000 & $1 \mathrm{a}$ \\
\hline $33 \mathrm{O}$ & $\mathrm{O} 32$ & 0.37899 & 0.16115 & 0.43564 & 1.000 & 1.000 & $1 \mathrm{a}$ \\
\hline $34 \mathrm{O}$ & $\mathrm{O} 33$ & 0.50494 & 0.25250 & 0.02237 & 1.000 & 1.000 & 1a \\
\hline $35 \mathrm{O}$ & O34 & 0.50498 & 0.00261 & 0.13931 & 1.000 & 1.000 & $1 \mathrm{a}$ \\
\hline $36 \mathrm{O}$ & $\mathrm{O} 35$ & 0.49892 & 0.25442 & 0.25936 & 1.000 & 1.000 & $1 \mathrm{a}$ \\
\hline $37 \mathrm{O}$ & O36 & 0.50510 & 0.00293 & 0.38257 & 1.000 & 1.000 & $1 \mathrm{a}$ \\
\hline $38 \mathrm{O}$ & $\mathrm{O} 37$ & 0.50494 & 0.25250 & 0.11620 & 1.000 & 1.000 & 1a \\
\hline $39 \mathrm{O}$ & $\mathrm{O} 38$ & 0.50502 & 0.00260 & 0.23442 & 1.000 & 1.000 & $1 \mathrm{a}$ \\
\hline $40 \mathrm{O}$ & O39 & 0.49847 & 0.25294 & 0.35641 & 1.000 & 1.000 & 1a \\
\hline $41 \mathrm{O}$ & $\mathrm{O} 40$ & 0.50465 & 0.00198 & 0.47609 & 1.000 & 1.000 & $1 \mathrm{a}$ \\
\hline $42 \mathrm{O}$ & O41 & 0.62994 & 0.10046 & 0.06928 & 1.000 & 1.000 & $1 \mathrm{a}$ \\
\hline $43 \mathrm{O}$ & $\mathrm{O} 42$ & 0.62999 & 0.35193 & 0.18834 & 1.000 & 1.000 & $1 \mathrm{a}$ \\
\hline $44 \mathrm{O}$ & $\mathrm{O} 43$ & 0.63032 & 0.09894 & 0.30894 & 1.000 & 1.000 & $1 \mathrm{a}$ \\
\hline $45 \mathrm{O}$ & $\mathrm{O} 44$ & 0.62795 & 0.35101 & 0.43406 & 1.000 & 1.000 & $1 \mathrm{a}$ \\
\hline $46 \mathrm{O}$ & O45 & 0.62994 & 0.40453 & 0.06928 & 1.000 & 1.000 & $1 \mathrm{a}$ \\
\hline $47 \mathrm{O}$ & O46 & 0.62995 & 0.15481 & 0.18870 & 1.000 & 1.000 & 1a \\
\hline $48 \mathrm{O}$ & O47 & 0.63030 & 0.40690 & 0.31007 & 1.000 & 1.000 & $1 \mathrm{a}$ \\
\hline $49 \mathrm{O}$ & O48 & 0.62974 & 0.16093 & 0.43454 & 1.000 & 1.000 & 1a \\
\hline $50 \mathrm{O}$ & O49 & 0.75494 & 0.25250 & 0.02237 & 1.000 & 1.000 & $1 \mathrm{a}$ \\
\hline $51 \mathrm{O}$ & O50 & 0.75500 & 0.00272 & 0.13926 & 1.000 & 1.000 & $1 \mathrm{a}$ \\
\hline $52 \mathrm{O}$ & O51 & 0.76110 & 0.25389 & 0.25939 & 1.000 & 1.000 & $1 \mathrm{a}$ \\
\hline $53 \mathrm{O}$ & O52 & 0.75471 & 0.00216 & 0.38251 & 1.000 & 1.000 & $1 \mathrm{a}$ \\
\hline $54 \mathrm{O}$ & O53 & 0.75494 & 0.25250 & 0.11620 & 1.000 & 1.000 & $1 \mathrm{a}$ \\
\hline $55 \mathrm{O}$ & O54 & 0.75528 & 0.00260 & 0.23442 & 1.000 & 1.000 & $1 \mathrm{a}$ \\
\hline $56 \mathrm{O}$ & O55 & 0.76148 & 0.25403 & 0.35678 & 1.000 & 1.000 & 1a \\
\hline $57 \mathrm{O}$ & O56 & 0.75451 & 0.00282 & 0.47616 & 1.000 & 1.000 & $1 \mathrm{a}$ \\
\hline $58 \mathrm{O}$ & O57 & 0.87994 & 0.10046 & 0.06928 & 1.000 & 1.000 & $1 \mathrm{a}$ \\
\hline $59 \mathrm{O}$ & O58 & 0.88079 & 0.35190 & 0.18765 & 1.000 & 1.000 & $1 \mathrm{a}$ \\
\hline $60 \mathrm{O}$ & O59 & 0.88022 & 0.10086 & 0.30886 & 1.000 & 1.000 & $1 \mathrm{a}$ \\
\hline $61 \mathrm{O}$ & O60 & 0.88117 & 0.34679 & 0.43606 & 1.000 & 1.000 & 1a \\
\hline $62 \mathrm{O}$ & O61 & 0.87994 & 0.40453 & 0.06928 & 1.000 & 1.000 & $1 \mathrm{a}$ \\
\hline $63 \mathrm{O}$ & O62 & 0.88078 & 0.15443 & 0.18790 & 1.000 & 1.000 & $1 \mathrm{a}$ \\
\hline $64 \mathrm{O}$ & O63 & 0.88091 & 0.40499 & 0.30823 & 1.000 & 1.000 & $1 \mathrm{a}$ \\
\hline $65 \mathrm{O}$ & O64 & 0.88019 & 0.15922 & 0.43455 & 1.000 & 1.000 & $1 \mathrm{a}$ \\
\hline $66 \mathrm{O}$ & O65 & 0.00495 & 0.75250 & 0.02237 & 1.000 & 1.000 & $1 \mathrm{a}$ \\
\hline $67 \mathrm{O}$ & O66 & 0.00502 & 0.50251 & 0.13926 & 1.000 & 1.000 & $1 \mathrm{a}$ \\
\hline $68 \mathrm{O}$ & O67 & 0.00532 & 0.75228 & 0.26105 & 1.000 & 1.000 & $1 \mathrm{a}$ \\
\hline $69 \mathrm{O}$ & O68 & 0.00460 & 0.50169 & 0.38485 & 1.000 & 1.000 & $1 \mathrm{a}$ \\
\hline $70 \mathrm{O}$ & O69 & 0.00495 & 0.75250 & 0.11620 & 1.000 & 1.000 & $1 \mathrm{a}$ \\
\hline $71 \mathrm{O}$ & $\mathrm{O} 70$ & 0.00503 & 0.50258 & 0.23423 & 1.000 & 1.000 & $1 \mathrm{a}$ \\
\hline
\end{tabular}




\begin{tabular}{|c|c|c|c|c|c|c|c|}
\hline $72 \mathrm{O}$ & O71 & 0.00310 & 0.75091 & 0.35485 & 1.000 & 1.000 & 1a \\
\hline $73 \mathrm{O}$ & O72 & 0.00483 & 0.50251 & 0.48015 & 1.000 & 1.000 & $1 \mathrm{a}$ \\
\hline $74 \mathrm{O}$ & $\mathrm{O} 73$ & 0.12994 & 0.60046 & 0.06928 & 1.000 & 1.000 & $1 \mathrm{a}$ \\
\hline $75 \mathrm{O}$ & $\mathrm{O} 74$ & 0.12925 & 0.85091 & 0.18788 & 1.000 & 1.000 & $1 \mathrm{a}$ \\
\hline $76 \mathrm{O}$ & $\mathrm{O} 75$ & 0.12911 & 0.59970 & 0.30848 & 1.000 & 1.000 & $1 \mathrm{a}$ \\
\hline $77 \mathrm{O}$ & O76 & 0.12761 & 0.84604 & 0.43353 & 1.000 & 1.000 & $1 \mathrm{a}$ \\
\hline $78 \mathrm{O}$ & O77 & 0.12994 & 0.90453 & 0.06928 & 1.000 & 1.000 & $1 \mathrm{a}$ \\
\hline $79 \mathrm{O}$ & $\mathrm{O} 78$ & 0.12938 & 0.65331 & 0.18760 & 1.000 & 1.000 & $1 \mathrm{a}$ \\
\hline $80 \mathrm{O}$ & O79 & 0.12993 & 0.90451 & 0.30896 & 1.000 & 1.000 & $1 \mathrm{a}$ \\
\hline $81 \mathrm{O}$ & O80 & 0.12651 & 0.65726 & 0.43495 & 1.000 & 1.000 & $1 \mathrm{a}$ \\
\hline $82 \mathrm{O}$ & O81 & 0.25495 & 0.75250 & 0.02237 & 1.000 & 1.000 & $1 \mathrm{a}$ \\
\hline $83 \mathrm{O}$ & O82 & 0.25502 & 0.50269 & 0.13925 & 1.000 & 1.000 & $1 \mathrm{a}$ \\
\hline $84 \mathrm{O}$ & O83 & 0.24891 & 0.75106 & 0.25947 & 1.000 & 1.000 & $1 \mathrm{a}$ \\
\hline $85 \mathrm{O}$ & O84 & 0.24503 & 0.50142 & 0.38059 & 1.000 & 1.000 & $1 \mathrm{a}$ \\
\hline $86 \mathrm{O}$ & O85 & 0.25495 & 0.75250 & 0.11620 & 1.000 & 1.000 & $1 \mathrm{a}$ \\
\hline $87 \mathrm{O}$ & O86 & 0.25511 & 0.50271 & 0.23431 & 1.000 & 1.000 & $1 \mathrm{a}$ \\
\hline $88 \mathrm{O}$ & O87 & 0.24819 & 0.75049 & 0.35757 & 1.000 & 1.000 & $1 \mathrm{a}$ \\
\hline $89 \mathrm{O}$ & O88 & 0.25956 & 0.50467 & 0.47391 & 1.000 & 1.000 & $1 \mathrm{a}$ \\
\hline $90 \mathrm{O}$ & O89 & 0.37995 & 0.60046 & 0.06928 & 1.000 & 1.000 & $1 \mathrm{a}$ \\
\hline $91 \mathrm{O}$ & O90 & 0.38005 & 0.85055 & 0.18864 & 1.000 & 1.000 & $1 \mathrm{a}$ \\
\hline $92 \mathrm{O}$ & O91 & 0.37966 & 0.59780 & 0.31019 & 1.000 & 1.000 & $1 \mathrm{a}$ \\
\hline $93 \mathrm{O}$ & O92 & 0.38158 & 0.84451 & 0.43298 & 1.000 & 1.000 & $1 \mathrm{a}$ \\
\hline $94 \mathrm{O}$ & O93 & 0.37995 & 0.90453 & 0.06928 & 1.000 & 1.000 & $1 \mathrm{a}$ \\
\hline $95 \mathrm{O}$ & O94 & 0.37992 & 0.65332 & 0.18830 & 1.000 & 1.000 & $1 \mathrm{a}$ \\
\hline $96 \mathrm{O}$ & O95 & 0.37977 & 0.90622 & 0.30903 & 1.000 & 1.000 & $1 \mathrm{a}$ \\
\hline $97 \mathrm{O}$ & O96 & 0.38339 & 0.65330 & 0.43364 & 1.000 & 1.000 & $1 \mathrm{a}$ \\
\hline $98 \mathrm{O}$ & O97 & 0.50494 & 0.75250 & 0.02237 & 1.000 & 1.000 & $1 \mathrm{a}$ \\
\hline $99 \mathrm{O}$ & O98 & 0.50500 & 0.50255 & 0.13952 & 1.000 & 1.000 & $1 \mathrm{a}$ \\
\hline $100 \mathrm{O}$ & O99 & 0.51039 & 0.75065 & 0.25936 & 1.000 & 1.000 & $1 \mathrm{a}$ \\
\hline $101 \mathrm{O}$ & O100 & 0.50482 & 0.50186 & 0.38654 & 1.000 & 1.000 & $1 \mathrm{a}$ \\
\hline $102 \mathrm{O}$ & O101 & 0.50494 & 0.75250 & 0.11620 & 1.000 & 1.000 & $1 \mathrm{a}$ \\
\hline $103 \mathrm{O}$ & O102 & 0.50498 & 0.50257 & 0.23508 & 1.000 & 1.000 & $1 \mathrm{a}$ \\
\hline $104 \mathrm{O}$ & $\mathrm{O} 103$ & 0.51214 & 0.75158 & 0.35576 & 1.000 & 1.000 & $1 \mathrm{a}$ \\
\hline $105 \mathrm{O}$ & O104 & 0.62994 & 0.60046 & 0.06928 & 1.000 & 1.000 & $1 \mathrm{a}$ \\
\hline $106 \mathrm{O}$ & O105 & 0.63077 & 0.85081 & 0.18804 & 1.000 & 1.000 & $1 \mathrm{a}$ \\
\hline $107 \mathrm{O}$ & O106 & 0.63052 & 0.59987 & 0.30961 & 1.000 & 1.000 & $1 \mathrm{a}$ \\
\hline $108 \mathrm{O}$ & O107 & 0.63059 & 0.84369 & 0.43511 & 1.000 & 1.000 & $1 \mathrm{a}$ \\
\hline $109 \mathrm{O}$ & O108 & 0.62994 & 0.90453 & 0.06928 & 1.000 & 1.000 & $1 \mathrm{a}$ \\
\hline $110 \mathrm{O}$ & O109 & 0.63092 & 0.65300 & 0.18769 & 1.000 & 1.000 & 1a \\
\hline $111 \mathrm{O}$ & O110 & 0.63040 & 0.90344 & 0.30861 & 1.000 & 1.000 & $1 \mathrm{a}$ \\
\hline $112 \mathrm{O}$ & O111 & 0.62899 & 0.65325 & 0.43433 & 1.000 & 1.000 & $1 \mathrm{a}$ \\
\hline $113 \mathrm{O}$ & $\mathrm{O} 112$ & 0.75494 & 0.75250 & 0.02237 & 1.000 & 1.000 & $1 \mathrm{a}$ \\
\hline $114 \mathrm{O}$ & O113 & 0.75492 & 0.50228 & 0.13925 & 1.000 & 1.000 & $1 \mathrm{a}$ \\
\hline $115 \mathrm{O}$ & O114 & 0.75594 & 0.75114 & 0.26082 & 1.000 & 1.000 & $1 \mathrm{a}$ \\
\hline
\end{tabular}




\begin{tabular}{|c|c|c|c|c|c|c|c|}
\hline $116 \mathrm{O}$ & $\mathrm{O} 115$ & 0.76488 & 0.50215 & 0.38054 & 1.000 & 1.000 & $1 \mathrm{a}$ \\
\hline $117 \mathrm{O}$ & O116 & 0.75494 & 0.75250 & 0.11620 & 1.000 & 1.000 & 1a \\
\hline $118 \mathrm{O}$ & $\mathrm{O} 117$ & 0.75491 & 0.50237 & 0.23427 & 1.000 & 1.000 & $1 \mathrm{a}$ \\
\hline 1190 & O118 & 0.75657 & 0.75091 & 0.35516 & 1.000 & 1.000 & $1 \mathrm{a}$ \\
\hline $120 \mathrm{O}$ & O119 & 0.74952 & 0.50167 & 0.47383 & 1.000 & 1.000 & $1 \mathrm{a}$ \\
\hline $121 \mathrm{O}$ & $\mathrm{O} 120$ & 0.87994 & 0.60046 & 0.06928 & 1.000 & 1.000 & 1a \\
\hline $122 \mathrm{O}$ & O121 & 0.88009 & 0.85056 & 0.18842 & 1.000 & 1.000 & 1a \\
\hline $123 \mathrm{O}$ & O122 & 0.88074 & 0.60047 & 0.30815 & 1.000 & 1.000 & 1a \\
\hline $124 \mathrm{O}$ & $\mathrm{O} 123$ & 0.87877 & 0.84551 & 0.43389 & 1.000 & 1.000 & $1 \mathrm{a}$ \\
\hline $125 \mathrm{O}$ & O124 & 0.87994 & 0.90453 & 0.06928 & 1.000 & 1.000 & la \\
\hline $126 \mathrm{O}$ & $\mathrm{O} 125$ & 0.87995 & 0.65354 & 0.18817 & 1.000 & 1.000 & $1 \mathrm{a}$ \\
\hline $127 \mathrm{O}$ & O126 & 0.88029 & 0.90361 & 0.30856 & 1.000 & 1.000 & $1 \mathrm{a}$ \\
\hline $128 \mathrm{O}$ & $\mathrm{O} 127$ & 0.87967 & 0.65753 & 0.43525 & 1.000 & 1.000 & $1 \mathrm{a}$ \\
\hline $129 \mathrm{O}$ & O128 & 0.24874 & 0.75272 & 0.55517 & 1.000 & 1.000 & 1a \\
\hline $130 \mathrm{Ti}$ & Ti1 & 0.00495 & 0.00250 & 0.06928 & 1.000 & 1.000 & $1 \mathrm{a}$ \\
\hline $131 \mathrm{Ti}$ & Ti2 & 0.00506 & 0.25233 & 0.18538 & 1.000 & 1.000 & 1a \\
\hline $132 \mathrm{Ti}$ & Ti3 & 0.00513 & 0.00262 & 0.31307 & 1.000 & 1.000 & 1a \\
\hline $133 \mathrm{Ti}$ & Ti4 & 0.00523 & 0.25235 & 0.42231 & 1.000 & 1.000 & $1 \mathrm{a}$ \\
\hline $134 \mathrm{Ti}$ & Ti5 & 0.12994 & 0.25250 & 0.06928 & 1.000 & 1.000 & $1 \mathrm{a}$ \\
\hline $135 \mathrm{Ti}$ & Ti6 & 0.12995 & 0.00244 & 0.18974 & 1.000 & 1.000 & $1 \mathrm{a}$ \\
\hline $136 \mathrm{Ti}$ & Ti7 & 0.12964 & 0.25165 & 0.30469 & 1.000 & 1.000 & $1 \mathrm{a}$ \\
\hline $137 \mathrm{Ti}$ & Ti8 & 0.12957 & 0.00269 & 0.43561 & 1.000 & 1.000 & 1a \\
\hline $138 \mathrm{Ti}$ & Ti9 & 0.25495 & 0.00250 & 0.06928 & 1.000 & 1.000 & $1 \mathrm{a}$ \\
\hline $139 \mathrm{Ti}$ & Ti10 & 0.25485 & 0.25286 & 0.18536 & 1.000 & 1.000 & $1 \mathrm{a}$ \\
\hline $140 \mathrm{Ti}$ & Til1 & 0.25570 & 0.00261 & 0.31310 & 1.000 & 1.000 & 1a \\
\hline $141 \mathrm{Ti}$ & Ti12 & 0.25441 & 0.25313 & 0.42251 & 1.000 & 1.000 & $1 \mathrm{a}$ \\
\hline $142 \mathrm{Ti}$ & Ti13 & 0.37995 & 0.25250 & 0.06928 & 1.000 & 1.000 & $1 \mathrm{a}$ \\
\hline $143 \mathrm{Ti}$ & Ti14 & 0.37999 & 0.00293 & 0.18983 & 1.000 & 1.000 & $1 \mathrm{a}$ \\
\hline $144 \mathrm{Ti}$ & Ti15 & 0.38212 & 0.25150 & 0.30384 & 1.000 & 1.000 & $1 \mathrm{a}$ \\
\hline $145 \mathrm{Ti}$ & Ti16 & 0.37992 & 0.00246 & 0.43574 & 1.000 & 1.000 & $1 \mathrm{a}$ \\
\hline $146 \mathrm{Ti}$ & Ti17 & 0.50494 & 0.00250 & 0.06928 & 1.000 & 1.000 & $1 \mathrm{a}$ \\
\hline $147 \mathrm{Ti}$ & Ti18 & 0.50508 & 0.25244 & 0.18600 & 1.000 & 1.000 & $1 \mathrm{a}$ \\
\hline $148 \mathrm{Ti}$ & Ti19 & 0.50514 & 0.00244 & 0.31307 & 1.000 & 1.000 & $1 \mathrm{a}$ \\
\hline $149 \mathrm{Ti}$ & Ti20 & 0.50417 & 0.25353 & 0.42262 & 1.000 & 1.000 & 1a \\
\hline $150 \mathrm{Ti}$ & Ti21 & 0.62994 & 0.25250 & 0.06928 & 1.000 & 1.000 & $1 \mathrm{a}$ \\
\hline $151 \mathrm{Ti}$ & Ti22 & 0.63006 & 0.00260 & 0.18981 & 1.000 & 1.000 & $1 \mathrm{a}$ \\
\hline $152 \mathrm{Ti}$ & Ti23 & 0.63010 & 0.25168 & 0.30676 & 1.000 & 1.000 & $1 \mathrm{a}$ \\
\hline $153 \mathrm{Ti}$ & Ti24 & 0.62975 & 0.00233 & 0.43591 & 1.000 & 1.000 & $1 \mathrm{a}$ \\
\hline $154 \mathrm{Ti}$ & Ti25 & 0.75494 & 0.00250 & 0.06928 & 1.000 & 1.000 & $1 \mathrm{a}$ \\
\hline $155 \mathrm{Ti}$ & Ti26 & 0.75499 & 0.25277 & 0.18600 & 1.000 & 1.000 & 1a \\
\hline $156 \mathrm{Ti}$ & Ti27 & 0.75455 & 0.00267 & 0.31312 & 1.000 & 1.000 & $1 \mathrm{a}$ \\
\hline $157 \mathrm{Ti}$ & Ti28 & 0.75599 & 0.25267 & 0.42316 & 1.000 & 1.000 & $1 \mathrm{a}$ \\
\hline $158 \mathrm{Ti}$ & Ti29 & 0.87994 & 0.25250 & 0.06928 & 1.000 & 1.000 & $1 \mathrm{a}$ \\
\hline $159 \mathrm{Ti}$ & Ti30 & 0.88012 & 0.00294 & 0.18977 & 1.000 & 1.000 & 1a \\
\hline
\end{tabular}




\begin{tabular}{|c|c|c|c|c|c|c|c|}
\hline $160 \mathrm{Ti}$ & Ti31 & 0.87776 & 0.25172 & 0.30395 & 1.000 & 1.000 & 1a \\
\hline $161 \mathrm{Ti}$ & Ti32 & 0.87955 & 0.00243 & 0.43594 & 1.000 & 1.000 & 1a \\
\hline $162 \mathrm{Ti}$ & Ti33 & 0.00495 & 0.50250 & 0.06928 & 1.000 & 1.000 & 1a \\
\hline $163 \mathrm{Ti}$ & Ti34 & 0.00511 & 0.75268 & 0.18552 & 1.000 & 1.000 & 1a \\
\hline $164 \mathrm{Ti}$ & Ti35 & 0.00501 & 0.50249 & 0.31441 & 1.000 & 1.000 & 1a \\
\hline $165 \mathrm{Ti}$ & Ti36 & 0.00367 & 0.75217 & 0.42161 & 1.000 & 1.000 & 1a \\
\hline $166 \mathrm{Ti}$ & Ti37 & 0.12994 & 0.75250 & 0.06928 & 1.000 & 1.000 & 1a \\
\hline $167 \mathrm{Ti}$ & Ti38 & 0.12814 & 0.50303 & 0.18899 & 1.000 & 1.000 & 1a \\
\hline $168 \mathrm{Ti}$ & Ti39 & 0.13364 & 0.75367 & 0.30480 & 1.000 & 1.000 & 1a \\
\hline $169 \mathrm{Ti}$ & Ti40 & 0.12547 & 0.50243 & 0.43816 & 1.000 & 1.000 & 1a \\
\hline $170 \mathrm{Ti}$ & Ti41 & 0.25495 & 0.50250 & 0.06928 & 1.000 & 1.000 & 1a \\
\hline $171 \mathrm{Ti}$ & Ti42 & 0.25496 & 0.75300 & 0.18604 & 1.000 & 1.000 & 1a \\
\hline $172 \mathrm{Ti}$ & Ti43 & 0.25361 & 0.50261 & 0.30642 & 1.000 & 1.000 & 1a \\
\hline $173 \mathrm{Ti}$ & Ti44 & 0.25343 & 0.75260 & 0.42542 & 1.000 & 1.000 & 1a \\
\hline $174 \mathrm{Ti}$ & Ti45 & 0.37995 & 0.75250 & 0.06928 & 1.000 & 1.000 & 1a \\
\hline $175 \mathrm{Ti}$ & Ti46 & 0.38047 & 0.50272 & 0.18833 & 1.000 & 1.000 & 1a \\
\hline $176 \mathrm{Ti}$ & Ti47 & 0.37903 & 0.75325 & 0.30720 & 1.000 & 1.000 & 1a \\
\hline $177 \mathrm{Ti}$ & Ti48 & 0.36140 & 0.50261 & 0.42319 & 1.000 & 1.000 & 1a \\
\hline $178 \mathrm{Ti}$ & Ti49 & 0.50494 & 0.50250 & 0.06928 & 1.000 & 1.000 & 1a \\
\hline $179 \mathrm{Ti}$ & Ti50 & 0.50489 & 0.75284 & 0.18605 & 1.000 & 1.000 & 1a \\
\hline $180 \mathrm{Ti}$ & Ti51 & 0.50500 & 0.50231 & 0.31035 & 1.000 & 1.000 & 1a \\
\hline $181 \mathrm{Ti}$ & Ti52 & 0.50601 & 0.75106 & 0.42181 & 1.000 & 1.000 & 1a \\
\hline $182 \mathrm{Ti}$ & Ti53 & 0.62994 & 0.75250 & 0.06928 & 1.000 & 1.000 & 1a \\
\hline $183 \mathrm{Ti}$ & Ti54 & 0.62960 & 0.50288 & 0.18832 & 1.000 & 1.000 & 1a \\
\hline $184 \mathrm{Ti}$ & Ti55 & 0.62800 & 0.75346 & 0.30341 & 1.000 & 1.000 & la \\
\hline $185 \mathrm{Ti}$ & Ti56 & 0.64818 & 0.50226 & 0.42302 & 1.000 & 1.000 & 1a \\
\hline $186 \mathrm{Ti}$ & Ti57 & 0.75494 & 0.50250 & 0.06928 & 1.000 & 1.000 & 1a \\
\hline $187 \mathrm{Ti}$ & Ti58 & 0.75518 & 0.75284 & 0.18528 & 1.000 & 1.000 & 1a \\
\hline $188 \mathrm{Ti}$ & Ti59 & 0.75644 & 0.50262 & 0.30638 & 1.000 & 1.000 & 1a \\
\hline $189 \mathrm{Ti}$ & Ti60 & 0.75527 & 0.75199 & 0.42197 & 1.000 & 1.000 & 1a \\
\hline $190 \mathrm{Ti}$ & Ti61 & 0.87994 & 0.75250 & 0.06928 & 1.000 & 1.000 & 1a \\
\hline $191 \mathrm{Ti}$ & Ti62 & 0.88205 & 0.50238 & 0.18898 & 1.000 & 1.000 & 1a \\
\hline $192 \mathrm{Ti}$ & Ti63 & 0.88098 & 0.75366 & 0.30410 & 1.000 & 1.000 & 1a \\
\hline $193 \mathrm{Ti}$ & Ti64 & 0.88357 & 0.50212 & 0.43845 & 1.000 & 1.000 & 1a \\
\hline
\end{tabular}


Title $\mathrm{CO} @ \mathrm{NNTi}_{5 \mathrm{c}}$ one surface and one subsurface EP

$\begin{array}{ll}\text { Lattice type } & \text { P } \\ \text { Space group name } & \text { P 1 } \\ \text { Space group number } & 1 \\ \text { Setting number } & 1\end{array}$

Lattice parameters

a $\quad b \quad$ c alpha beta gamma

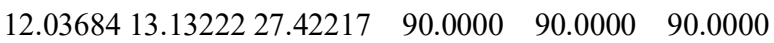

Unit-cell volume $=4334.634578 \AA \wedge 3$

Structure parameters

\begin{tabular}{|c|c|c|c|c|c|c|c|c|}
\hline & & $\mathrm{x}$ & $\mathrm{y}$ & z & Occ. & B & Site & Sym. \\
\hline $1 \mathrm{C}$ & $\mathrm{C} 1$ & 0.25218 & 0.75351 & 0.51514 & 1.000 & 1.000 & $1 \mathrm{a}$ & 1 \\
\hline $2 \mathrm{O}$ & $\mathrm{O} 1$ & 0.00495 & 0.25250 & 0.02237 & 1.000 & 1.000 & $1 \mathrm{a}$ & 1 \\
\hline 30 & $\mathrm{O} 2$ & 0.00508 & 0.00218 & 0.13921 & 1.000 & 1.000 & $1 \mathrm{a}$ & 1 \\
\hline $4 \mathrm{O}$ & $\mathrm{O} 3$ & 0.00568 & 0.25267 & 0.26101 & 1.000 & 1.000 & $1 \mathrm{a}$ & 1 \\
\hline $5 \mathrm{O}$ & $\mathrm{O} 4$ & 0.00333 & 0.00478 & 0.38199 & 1.000 & 1.000 & $1 \mathrm{a}$ & 1 \\
\hline 60 & O5 & 0.00495 & 0.25250 & 0.11620 & 1.000 & 1.000 & $1 \mathrm{a}$ & 1 \\
\hline $7 \mathrm{O}$ & O6 & 0.00501 & 0.00273 & 0.23436 & 1.000 & 1.000 & $1 \mathrm{a}$ & 1 \\
\hline $8 \mathrm{O}$ & $\mathrm{O} 7$ & 0.00613 & 0.25399 & 0.35516 & 1.000 & 1.000 & $1 \mathrm{a}$ & 1 \\
\hline $9 \mathrm{O}$ & O8 & 0.00383 & 0.00438 & 0.47604 & 1.000 & 1.000 & $1 \mathrm{a}$ & 1 \\
\hline $10 \mathrm{O}$ & O9 & 0.12994 & 0.10046 & 0.06928 & 1.000 & 1.000 & $1 \mathrm{a}$ & 1 \\
\hline $11 \mathrm{O}$ & $\mathrm{O} 10$ & 0.13005 & 0.35169 & 0.18815 & 1.000 & 1.000 & $1 \mathrm{a}$ & 1 \\
\hline $12 \mathrm{O}$ & O11 & 0.13014 & 0.10145 & 0.30860 & 1.000 & 1.000 & $1 \mathrm{a}$ & 1 \\
\hline $13 \mathrm{O}$ & $\mathrm{O} 12$ & 0.12960 & 0.34743 & 0.43530 & 1.000 & 1.000 & $1 \mathrm{a}$ & 1 \\
\hline $14 \mathrm{O}$ & $\mathrm{O} 13$ & 0.12994 & 0.40453 & 0.06928 & 1.000 & 1.000 & $1 \mathrm{a}$ & 1 \\
\hline $15 \mathrm{O}$ & $\mathrm{O} 14$ & 0.13005 & 0.15470 & 0.18834 & 1.000 & 1.000 & $1 \mathrm{a}$ & 1 \\
\hline $16 \mathrm{O}$ & O15 & 0.12943 & 0.40473 & 0.30812 & 1.000 & 1.000 & $1 \mathrm{a}$ & 1 \\
\hline $17 \mathrm{O}$ & O16 & 0.13087 & 0.15929 & 0.43408 & 1.000 & 1.000 & $1 \mathrm{a}$ & 1 \\
\hline $18 \mathrm{O}$ & $\mathrm{O} 17$ & 0.25495 & 0.25250 & 0.02237 & 1.000 & 1.000 & $1 \mathrm{a}$ & 1 \\
\hline $19 \mathrm{O}$ & O18 & 0.25481 & 0.00230 & 0.13921 & 1.000 & 1.000 & $1 \mathrm{a}$ & 1 \\
\hline $20 \mathrm{O}$ & O19 & 0.25405 & 0.25369 & 0.26110 & 1.000 & 1.000 & $1 \mathrm{a}$ & 1 \\
\hline $21 \mathrm{O}$ & $\mathrm{O} 20$ & 0.25538 & 0.00252 & 0.38227 & 1.000 & 1.000 & $1 \mathrm{a}$ & 1 \\
\hline $22 \mathrm{O}$ & $\mathrm{O} 21$ & 0.25495 & 0.25250 & 0.11620 & 1.000 & 1.000 & $1 \mathrm{a}$ & 1 \\
\hline $23 \mathrm{O}$ & $\mathrm{O} 22$ & 0.25482 & 0.00247 & 0.23439 & 1.000 & 1.000 & $1 \mathrm{a}$ & 1 \\
\hline $24 \mathrm{O}$ & $\mathrm{O} 23$ & 0.25352 & 0.25409 & 0.35547 & 1.000 & 1.000 & $1 \mathrm{a}$ & 1 \\
\hline $25 \mathrm{O}$ & $\mathrm{O} 24$ & 0.25432 & 0.99638 & 0.47576 & 1.000 & 1.000 & $1 \mathrm{a}$ & 1 \\
\hline $26 \mathrm{O}$ & $\mathrm{O} 25$ & 0.37995 & 0.10046 & 0.06928 & 1.000 & 1.000 & $1 \mathrm{a}$ & 1 \\
\hline $27 \mathrm{O}$ & $\mathrm{O} 26$ & 0.37901 & 0.35221 & 0.18753 & 1.000 & 1.000 & $1 \mathrm{a}$ & 1 \\
\hline
\end{tabular}




\begin{tabular}{|c|c|c|c|c|c|c|c|}
\hline $28 \mathrm{O}$ & $\mathrm{O} 27$ & 0.37953 & 0.10082 & 0.30855 & 1.000 & 1.000 & $1 \mathrm{a}$ \\
\hline $29 \mathrm{O}$ & $\mathrm{O} 28$ & 0.38106 & 0.35166 & 0.43511 & 1.000 & 1.000 & $1 \mathrm{a}$ \\
\hline $30 \mathrm{O}$ & $\mathrm{O} 29$ & 0.37995 & 0.40453 & 0.06928 & 1.000 & 1.000 & $1 \mathrm{a}$ \\
\hline $31 \mathrm{O}$ & $\mathrm{O} 30$ & 0.37909 & 0.15447 & 0.18787 & 1.000 & 1.000 & 1a \\
\hline $32 \mathrm{O}$ & $\mathrm{O} 31$ & 0.37906 & 0.40545 & 0.30940 & 1.000 & 1.000 & $1 \mathrm{a}$ \\
\hline $33 \mathrm{O}$ & $\mathrm{O} 32$ & 0.37843 & 0.16075 & 0.43554 & 1.000 & 1.000 & $1 \mathrm{a}$ \\
\hline $34 \mathrm{O}$ & $\mathrm{O} 33$ & 0.50494 & 0.25250 & 0.02237 & 1.000 & 1.000 & 1a \\
\hline $35 \mathrm{O}$ & O34 & 0.50502 & 0.00244 & 0.13926 & 1.000 & 1.000 & $1 \mathrm{a}$ \\
\hline $36 \mathrm{O}$ & $\mathrm{O} 35$ & 0.49838 & 0.25420 & 0.25930 & 1.000 & 1.000 & $1 \mathrm{a}$ \\
\hline $37 \mathrm{O}$ & O36 & 0.50477 & 0.00275 & 0.38233 & 1.000 & 1.000 & $1 \mathrm{a}$ \\
\hline $38 \mathrm{O}$ & $\mathrm{O} 37$ & 0.50494 & 0.25250 & 0.11620 & 1.000 & 1.000 & 1a \\
\hline $39 \mathrm{O}$ & $\mathrm{O} 38$ & 0.50473 & 0.00264 & 0.23432 & 1.000 & 1.000 & $1 \mathrm{a}$ \\
\hline $40 \mathrm{O}$ & O39 & 0.49821 & 0.25296 & 0.35623 & 1.000 & 1.000 & 1a \\
\hline $41 \mathrm{O}$ & $\mathrm{O} 40$ & 0.50485 & 0.00348 & 0.47619 & 1.000 & 1.000 & $1 \mathrm{a}$ \\
\hline $42 \mathrm{O}$ & O41 & 0.62994 & 0.10046 & 0.06928 & 1.000 & 1.000 & $1 \mathrm{a}$ \\
\hline $43 \mathrm{O}$ & $\mathrm{O} 42$ & 0.62992 & 0.35188 & 0.18831 & 1.000 & 1.000 & $1 \mathrm{a}$ \\
\hline $44 \mathrm{O}$ & $\mathrm{O} 43$ & 0.62989 & 0.09867 & 0.30863 & 1.000 & 1.000 & $1 \mathrm{a}$ \\
\hline $45 \mathrm{O}$ & $\mathrm{O} 44$ & 0.62739 & 0.35070 & 0.43408 & 1.000 & 1.000 & $1 \mathrm{a}$ \\
\hline $46 \mathrm{O}$ & O45 & 0.62994 & 0.40453 & 0.06928 & 1.000 & 1.000 & $1 \mathrm{a}$ \\
\hline $47 \mathrm{O}$ & O46 & 0.62990 & 0.15474 & 0.18866 & 1.000 & 1.000 & 1a \\
\hline $48 \mathrm{O}$ & O47 & 0.63041 & 0.40701 & 0.30925 & 1.000 & 1.000 & 1a \\
\hline $49 \mathrm{O}$ & O48 & 0.62958 & 0.16093 & 0.43411 & 1.000 & 1.000 & 1a \\
\hline $50 \mathrm{O}$ & O49 & 0.75494 & 0.25250 & 0.02237 & 1.000 & 1.000 & $1 \mathrm{a}$ \\
\hline $51 \mathrm{O}$ & O50 & 0.75479 & 0.00230 & 0.13925 & 1.000 & 1.000 & $1 \mathrm{a}$ \\
\hline $52 \mathrm{O}$ & O51 & 0.76106 & 0.25383 & 0.25924 & 1.000 & 1.000 & 1a \\
\hline $53 \mathrm{O}$ & O52 & 0.75409 & 0.00274 & 0.38225 & 1.000 & 1.000 & 1a \\
\hline $54 \mathrm{O}$ & O53 & 0.75494 & 0.25250 & 0.11620 & 1.000 & 1.000 & $1 \mathrm{a}$ \\
\hline $55 \mathrm{O}$ & O54 & 0.75507 & 0.00238 & 0.23427 & 1.000 & 1.000 & $1 \mathrm{a}$ \\
\hline $56 \mathrm{O}$ & O55 & 0.76125 & 0.25428 & 0.35646 & 1.000 & 1.000 & 1a \\
\hline $57 \mathrm{O}$ & O56 & 0.75390 & 0.00354 & 0.47619 & 1.000 & 1.000 & $1 \mathrm{a}$ \\
\hline $58 \mathrm{O}$ & O57 & 0.87994 & 0.10046 & 0.06928 & 1.000 & 1.000 & $1 \mathrm{a}$ \\
\hline $59 \mathrm{O}$ & O58 & 0.88081 & 0.35199 & 0.18754 & 1.000 & 1.000 & $1 \mathrm{a}$ \\
\hline $60 \mathrm{O}$ & O59 & 0.87962 & 0.10087 & 0.30838 & 1.000 & 1.000 & $1 \mathrm{a}$ \\
\hline $61 \mathrm{O}$ & O60 & 0.88111 & 0.34693 & 0.43567 & 1.000 & 1.000 & 1a \\
\hline $62 \mathrm{O}$ & O61 & 0.87994 & 0.40453 & 0.06928 & 1.000 & 1.000 & $1 \mathrm{a}$ \\
\hline $63 \mathrm{O}$ & O62 & 0.88078 & 0.15436 & 0.18778 & 1.000 & 1.000 & $1 \mathrm{a}$ \\
\hline $64 \mathrm{O}$ & O63 & 0.88083 & 0.40515 & 0.30779 & 1.000 & 1.000 & $1 \mathrm{a}$ \\
\hline $65 \mathrm{O}$ & O64 & 0.87963 & 0.15969 & 0.43431 & 1.000 & 1.000 & $1 \mathrm{a}$ \\
\hline $66 \mathrm{O}$ & O65 & 0.00495 & 0.75250 & 0.02237 & 1.000 & 1.000 & $1 \mathrm{a}$ \\
\hline $67 \mathrm{O}$ & O66 & 0.00518 & 0.50302 & 0.13924 & 1.000 & 1.000 & $1 \mathrm{a}$ \\
\hline $68 \mathrm{O}$ & O67 & 0.00646 & 0.75250 & 0.26099 & 1.000 & 1.000 & $1 \mathrm{a}$ \\
\hline $69 \mathrm{O}$ & O68 & 0.00401 & 0.50183 & 0.38445 & 1.000 & 1.000 & $1 \mathrm{a}$ \\
\hline $70 \mathrm{O}$ & O69 & 0.00495 & 0.75250 & 0.11620 & 1.000 & 1.000 & $1 \mathrm{a}$ \\
\hline $71 \mathrm{O}$ & $\mathrm{O} 70$ & 0.00500 & 0.50265 & 0.23407 & 1.000 & 1.000 & $1 \mathrm{a}$ \\
\hline
\end{tabular}




\begin{tabular}{|c|c|c|c|c|c|c|c|}
\hline $72 \mathrm{O}$ & O71 & 0.00329 & 0.75018 & 0.35402 & 1.000 & 1.000 & 1a \\
\hline $73 \mathrm{O}$ & O72 & 0.00423 & 0.50179 & 0.47996 & 1.000 & 1.000 & $1 \mathrm{a}$ \\
\hline $74 \mathrm{O}$ & $\mathrm{O} 73$ & 0.12994 & 0.60046 & 0.06928 & 1.000 & 1.000 & $1 \mathrm{a}$ \\
\hline $75 \mathrm{O}$ & $\mathrm{O} 74$ & 0.13011 & 0.85059 & 0.18839 & 1.000 & 1.000 & $1 \mathrm{a}$ \\
\hline $76 \mathrm{O}$ & $\mathrm{O} 75$ & 0.12893 & 0.60006 & 0.30810 & 1.000 & 1.000 & $1 \mathrm{a}$ \\
\hline $77 \mathrm{O}$ & O76 & 0.11946 & 0.84850 & 0.43221 & 1.000 & 1.000 & $1 \mathrm{a}$ \\
\hline $78 \mathrm{O}$ & O77 & 0.12994 & 0.90453 & 0.06928 & 1.000 & 1.000 & $1 \mathrm{a}$ \\
\hline $79 \mathrm{O}$ & $\mathrm{O} 78$ & 0.13027 & 0.65378 & 0.18821 & 1.000 & 1.000 & $1 \mathrm{a}$ \\
\hline $80 \mathrm{O}$ & O79 & 0.12938 & 0.90439 & 0.30888 & 1.000 & 1.000 & $1 \mathrm{a}$ \\
\hline $81 \mathrm{O}$ & O80 & 0.12170 & 0.65711 & 0.43530 & 1.000 & 1.000 & $1 \mathrm{a}$ \\
\hline $82 \mathrm{O}$ & O81 & 0.25495 & 0.75250 & 0.02237 & 1.000 & 1.000 & $1 \mathrm{a}$ \\
\hline $83 \mathrm{O}$ & O82 & 0.25484 & 0.50284 & 0.13919 & 1.000 & 1.000 & $1 \mathrm{a}$ \\
\hline $84 \mathrm{O}$ & O83 & 0.25478 & 0.75155 & 0.26112 & 1.000 & 1.000 & $1 \mathrm{a}$ \\
\hline $85 \mathrm{O}$ & O84 & 0.24587 & 0.50385 & 0.38043 & 1.000 & 1.000 & $1 \mathrm{a}$ \\
\hline $86 \mathrm{O}$ & O85 & 0.25495 & 0.75250 & 0.11620 & 1.000 & 1.000 & $1 \mathrm{a}$ \\
\hline $87 \mathrm{O}$ & O86 & 0.25511 & 0.50275 & 0.23428 & 1.000 & 1.000 & $1 \mathrm{a}$ \\
\hline $88 \mathrm{O}$ & O87 & 0.25517 & 0.75087 & 0.35477 & 1.000 & 1.000 & $1 \mathrm{a}$ \\
\hline $89 \mathrm{O}$ & O88 & 0.25878 & 0.50615 & 0.47369 & 1.000 & 1.000 & $1 \mathrm{a}$ \\
\hline $90 \mathrm{O}$ & O89 & 0.37995 & 0.60046 & 0.06928 & 1.000 & 1.000 & $1 \mathrm{a}$ \\
\hline $91 \mathrm{O}$ & O90 & 0.37997 & 0.85048 & 0.18846 & 1.000 & 1.000 & $1 \mathrm{a}$ \\
\hline $92 \mathrm{O}$ & O91 & 0.38027 & 0.59949 & 0.30933 & 1.000 & 1.000 & $1 \mathrm{a}$ \\
\hline $93 \mathrm{O}$ & O92 & 0.38760 & 0.84418 & 0.43409 & 1.000 & 1.000 & $1 \mathrm{a}$ \\
\hline $94 \mathrm{O}$ & O93 & 0.37995 & 0.90453 & 0.06928 & 1.000 & 1.000 & $1 \mathrm{a}$ \\
\hline $95 \mathrm{O}$ & O94 & 0.37977 & 0.65351 & 0.18820 & 1.000 & 1.000 & $1 \mathrm{a}$ \\
\hline $96 \mathrm{O}$ & O95 & 0.38007 & 0.90401 & 0.30847 & 1.000 & 1.000 & $1 \mathrm{a}$ \\
\hline $97 \mathrm{O}$ & O96 & 0.39391 & 0.64900 & 0.43476 & 1.000 & 1.000 & $1 \mathrm{a}$ \\
\hline $98 \mathrm{O}$ & O97 & 0.50494 & 0.75250 & 0.02237 & 1.000 & 1.000 & $1 \mathrm{a}$ \\
\hline $99 \mathrm{O}$ & O98 & 0.50498 & 0.50275 & 0.13945 & 1.000 & 1.000 & $1 \mathrm{a}$ \\
\hline $100 \mathrm{O}$ & O99 & 0.50354 & 0.75101 & 0.26107 & 1.000 & 1.000 & $1 \mathrm{a}$ \\
\hline $101 \mathrm{O}$ & O100 & 0.50553 & 0.49961 & 0.38536 & 1.000 & 1.000 & $1 \mathrm{a}$ \\
\hline $102 \mathrm{O}$ & O101 & 0.50494 & 0.75250 & 0.11620 & 1.000 & 1.000 & $1 \mathrm{a}$ \\
\hline $103 \mathrm{O}$ & O102 & 0.50466 & 0.50237 & 0.23483 & 1.000 & 1.000 & $1 \mathrm{a}$ \\
\hline $104 \mathrm{O}$ & $\mathrm{O} 103$ & 0.50593 & 0.75151 & 0.35414 & 1.000 & 1.000 & $1 \mathrm{a}$ \\
\hline $105 \mathrm{O}$ & O104 & 0.62994 & 0.60046 & 0.06928 & 1.000 & 1.000 & $1 \mathrm{a}$ \\
\hline $106 \mathrm{O}$ & O105 & 0.62979 & 0.85037 & 0.18863 & 1.000 & 1.000 & $1 \mathrm{a}$ \\
\hline $107 \mathrm{O}$ & O106 & 0.63008 & 0.60043 & 0.30919 & 1.000 & 1.000 & $1 \mathrm{a}$ \\
\hline $108 \mathrm{O}$ & O107 & 0.63115 & 0.84378 & 0.43452 & 1.000 & 1.000 & $1 \mathrm{a}$ \\
\hline $109 \mathrm{O}$ & O108 & 0.62994 & 0.90453 & 0.06928 & 1.000 & 1.000 & $1 \mathrm{a}$ \\
\hline $110 \mathrm{O}$ & O109 & 0.62989 & 0.65347 & 0.18832 & 1.000 & 1.000 & 1a \\
\hline $111 \mathrm{O}$ & O110 & 0.62985 & 0.90289 & 0.30835 & 1.000 & 1.000 & $1 \mathrm{a}$ \\
\hline $112 \mathrm{O}$ & O111 & 0.63114 & 0.65281 & 0.43283 & 1.000 & 1.000 & $1 \mathrm{a}$ \\
\hline $113 \mathrm{O}$ & $\mathrm{O} 112$ & 0.75494 & 0.75250 & 0.02237 & 1.000 & 1.000 & $1 \mathrm{a}$ \\
\hline $114 \mathrm{O}$ & O113 & 0.75466 & 0.50277 & 0.13922 & 1.000 & 1.000 & $1 \mathrm{a}$ \\
\hline $115 \mathrm{O}$ & O114 & 0.75467 & 0.75124 & 0.26054 & 1.000 & 1.000 & $1 \mathrm{a}$ \\
\hline
\end{tabular}




\begin{tabular}{|c|c|c|c|c|c|c|c|}
\hline $116 \mathrm{O}$ & $\mathrm{O} 115$ & 0.76457 & 0.50115 & 0.38017 & 1.000 & 1.000 & $1 \mathrm{a}$ \\
\hline $117 \mathrm{O}$ & O116 & 0.75494 & 0.75250 & 0.11620 & 1.000 & 1.000 & 1a \\
\hline $118 \mathrm{O}$ & $\mathrm{O} 117$ & 0.75485 & 0.50262 & 0.23406 & 1.000 & 1.000 & $1 \mathrm{a}$ \\
\hline 1190 & O118 & 0.75509 & 0.75131 & 0.35413 & 1.000 & 1.000 & 1a \\
\hline $120 \mathrm{O}$ & O119 & 0.74908 & 0.50088 & 0.47368 & 1.000 & 1.000 & $1 \mathrm{a}$ \\
\hline $121 \mathrm{O}$ & $\mathrm{O} 120$ & 0.87994 & 0.60046 & 0.06928 & 1.000 & 1.000 & 1a \\
\hline $122 \mathrm{O}$ & O121 & 0.87999 & 0.85043 & 0.18852 & 1.000 & 1.000 & 1a \\
\hline $123 \mathrm{O}$ & O122 & 0.88039 & 0.60065 & 0.30772 & 1.000 & 1.000 & 1a \\
\hline $124 \mathrm{O}$ & $\mathrm{O} 123$ & 0.87716 & 0.84645 & 0.43337 & 1.000 & 1.000 & $1 \mathrm{a}$ \\
\hline $125 \mathrm{O}$ & O124 & 0.87994 & 0.90453 & 0.06928 & 1.000 & 1.000 & $1 \mathrm{a}$ \\
\hline $126 \mathrm{O}$ & $\mathrm{O} 125$ & 0.87990 & 0.65379 & 0.18827 & 1.000 & 1.000 & $1 \mathrm{a}$ \\
\hline $127 \mathrm{O}$ & O126 & 0.88001 & 0.90360 & 0.30836 & 1.000 & 1.000 & $1 \mathrm{a}$ \\
\hline $128 \mathrm{O}$ & $\mathrm{O} 127$ & 0.87761 & 0.65750 & 0.43495 & 1.000 & 1.000 & $1 \mathrm{a}$ \\
\hline $129 \mathrm{O}$ & O128 & 0.24966 & 0.75353 & 0.55665 & 1.000 & 1.000 & 1a \\
\hline $130 \mathrm{Ti}$ & Ti1 & 0.00495 & 0.00250 & 0.06928 & 1.000 & 1.000 & $1 \mathrm{a}$ \\
\hline $131 \mathrm{Ti}$ & Ti2 & 0.00501 & 0.25235 & 0.18540 & 1.000 & 1.000 & 1a \\
\hline $132 \mathrm{Ti}$ & Ti3 & 0.00468 & 0.00286 & 0.31317 & 1.000 & 1.000 & 1a \\
\hline $133 \mathrm{Ti}$ & Ti4 & 0.00540 & 0.25247 & 0.42225 & 1.000 & 1.000 & $1 \mathrm{a}$ \\
\hline $134 \mathrm{Ti}$ & Ti5 & 0.12994 & 0.25250 & 0.06928 & 1.000 & 1.000 & $1 \mathrm{a}$ \\
\hline $135 \mathrm{Ti}$ & Ti6 & 0.12994 & 0.00274 & 0.18983 & 1.000 & 1.000 & $1 \mathrm{a}$ \\
\hline $136 \mathrm{Ti}$ & Ti7 & 0.12965 & 0.25180 & 0.30474 & 1.000 & 1.000 & $1 \mathrm{a}$ \\
\hline $137 \mathrm{Ti}$ & Ti8 & 0.12837 & 0.00073 & 0.43551 & 1.000 & 1.000 & 1a \\
\hline $138 \mathrm{Ti}$ & Ti9 & 0.25495 & 0.00250 & 0.06928 & 1.000 & 1.000 & $1 \mathrm{a}$ \\
\hline $139 \mathrm{Ti}$ & Ti10 & 0.25494 & 0.25286 & 0.18535 & 1.000 & 1.000 & $1 \mathrm{a}$ \\
\hline $140 \mathrm{Ti}$ & Til1 & 0.25504 & 0.00246 & 0.31331 & 1.000 & 1.000 & 1a \\
\hline $141 \mathrm{Ti}$ & Ti12 & 0.25407 & 0.25294 & 0.42255 & 1.000 & 1.000 & $1 \mathrm{a}$ \\
\hline $142 \mathrm{Ti}$ & Ti13 & 0.37995 & 0.25250 & 0.06928 & 1.000 & 1.000 & $1 \mathrm{a}$ \\
\hline $143 \mathrm{Ti}$ & Ti14 & 0.37990 & 0.00304 & 0.18981 & 1.000 & 1.000 & $1 \mathrm{a}$ \\
\hline $144 \mathrm{Ti}$ & Ti15 & 0.38183 & 0.25136 & 0.30397 & 1.000 & 1.000 & $1 \mathrm{a}$ \\
\hline $145 \mathrm{Ti}$ & Ti16 & 0.38002 & 0.00046 & 0.43595 & 1.000 & 1.000 & $1 \mathrm{a}$ \\
\hline $146 \mathrm{Ti}$ & Ti17 & 0.50494 & 0.00250 & 0.06928 & 1.000 & 1.000 & $1 \mathrm{a}$ \\
\hline $147 \mathrm{Ti}$ & Ti18 & 0.50506 & 0.25238 & 0.18601 & 1.000 & 1.000 & $1 \mathrm{a}$ \\
\hline $148 \mathrm{Ti}$ & Ti19 & 0.50530 & 0.00262 & 0.31319 & 1.000 & 1.000 & $1 \mathrm{a}$ \\
\hline $149 \mathrm{Ti}$ & Ti20 & 0.50364 & 0.25305 & 0.42264 & 1.000 & 1.000 & 1a \\
\hline $150 \mathrm{Ti}$ & Ti21 & 0.62994 & 0.25250 & 0.06928 & 1.000 & 1.000 & $1 \mathrm{a}$ \\
\hline $151 \mathrm{Ti}$ & Ti22 & 0.62990 & 0.00277 & 0.18987 & 1.000 & 1.000 & $1 \mathrm{a}$ \\
\hline $152 \mathrm{Ti}$ & Ti23 & 0.62990 & 0.25175 & 0.30660 & 1.000 & 1.000 & $1 \mathrm{a}$ \\
\hline $153 \mathrm{Ti}$ & Ti24 & 0.62950 & 0.00224 & 0.43595 & 1.000 & 1.000 & $1 \mathrm{a}$ \\
\hline $154 \mathrm{Ti}$ & Ti25 & 0.75494 & 0.00250 & 0.06928 & 1.000 & 1.000 & $1 \mathrm{a}$ \\
\hline $155 \mathrm{Ti}$ & Ti26 & 0.75509 & 0.25279 & 0.18602 & 1.000 & 1.000 & 1a \\
\hline $156 \mathrm{Ti}$ & Ti27 & 0.75456 & 0.00264 & 0.31319 & 1.000 & 1.000 & $1 \mathrm{a}$ \\
\hline $157 \mathrm{Ti}$ & Ti28 & 0.75585 & 0.25301 & 0.42299 & 1.000 & 1.000 & $1 \mathrm{a}$ \\
\hline $158 \mathrm{Ti}$ & Ti29 & 0.87994 & 0.25250 & 0.06928 & 1.000 & 1.000 & $1 \mathrm{a}$ \\
\hline $159 \mathrm{Ti}$ & Ti30 & 0.88006 & 0.00293 & 0.18978 & 1.000 & 1.000 & 1a \\
\hline
\end{tabular}




\begin{tabular}{|c|c|c|c|c|c|c|c|}
\hline $160 \mathrm{Ti}$ & Ti31 & 0.87768 & 0.25177 & 0.30394 & 1.000 & 1.000 & 1a \\
\hline $161 \mathrm{Ti}$ & Ti32 & 0.87896 & 0.00291 & 0.43600 & 1.000 & 1.000 & 1a \\
\hline $162 \mathrm{Ti}$ & Ti33 & 0.00495 & 0.50250 & 0.06928 & 1.000 & 1.000 & 1a \\
\hline $163 \mathrm{Ti}$ & Ti34 & 0.00529 & 0.75278 & 0.18591 & 1.000 & 1.000 & 1a \\
\hline $164 \mathrm{Ti}$ & Ti35 & 0.00476 & 0.50259 & 0.31436 & 1.000 & 1.000 & 1a \\
\hline $165 \mathrm{Ti}$ & Ti36 & 0.00351 & 0.75326 & 0.42163 & 1.000 & 1.000 & 1a \\
\hline $166 \mathrm{Ti}$ & Ti37 & 0.12994 & 0.75250 & 0.06928 & 1.000 & 1.000 & 1a \\
\hline $167 \mathrm{Ti}$ & Ti38 & 0.12822 & 0.50276 & 0.18908 & 1.000 & 1.000 & 1a \\
\hline $168 \mathrm{Ti}$ & Ti39 & 0.13367 & 0.75354 & 0.30728 & 1.000 & 1.000 & 1a \\
\hline $169 \mathrm{Ti}$ & Ti40 & 0.12492 & 0.50389 & 0.43789 & 1.000 & 1.000 & 1a \\
\hline $170 \mathrm{Ti}$ & Ti41 & 0.25495 & 0.50250 & 0.06928 & 1.000 & 1.000 & 1a \\
\hline $171 \mathrm{Ti}$ & Ti42 & 0.25498 & 0.75300 & 0.18564 & 1.000 & 1.000 & 1a \\
\hline $172 \mathrm{Ti}$ & Ti43 & 0.25350 & 0.50296 & 0.30670 & 1.000 & 1.000 & 1a \\
\hline $173 \mathrm{Ti}$ & Ti44 & 0.25470 & 0.75167 & 0.43111 & 1.000 & 1.000 & 1a \\
\hline $174 \mathrm{Ti}$ & Ti45 & 0.37995 & 0.75250 & 0.06928 & 1.000 & 1.000 & 1a \\
\hline $175 \mathrm{Ti}$ & Ti46 & 0.38041 & 0.50250 & 0.18844 & 1.000 & 1.000 & 1a \\
\hline $176 \mathrm{Ti}$ & Ti47 & 0.37589 & 0.75370 & 0.30706 & 1.000 & 1.000 & 1a \\
\hline $177 \mathrm{Ti}$ & Ti48 & 0.36222 & 0.50319 & 0.42353 & 1.000 & 1.000 & 1a \\
\hline $178 \mathrm{Ti}$ & Ti49 & 0.50494 & 0.50250 & 0.06928 & 1.000 & 1.000 & 1a \\
\hline $179 \mathrm{Ti}$ & Ti50 & 0.50467 & 0.75275 & 0.18588 & 1.000 & 1.000 & 1a \\
\hline $180 \mathrm{Ti}$ & Ti51 & 0.50505 & 0.50209 & 0.31095 & 1.000 & 1.000 & 1a \\
\hline $181 \mathrm{Ti}$ & Ti52 & 0.50632 & 0.74993 & 0.42151 & 1.000 & 1.000 & 1a \\
\hline $182 \mathrm{Ti}$ & Ti53 & 0.62994 & 0.75250 & 0.06928 & 1.000 & 1.000 & 1a \\
\hline $183 \mathrm{Ti}$ & Ti54 & 0.62908 & 0.50257 & 0.18854 & 1.000 & 1.000 & 1a \\
\hline $184 \mathrm{Ti}$ & Ti55 & 0.62841 & 0.75344 & 0.30355 & 1.000 & 1.000 & la \\
\hline $185 \mathrm{Ti}$ & Ti56 & 0.64819 & 0.50185 & 0.42283 & 1.000 & 1.000 & 1a \\
\hline $186 \mathrm{Ti}$ & Ti57 & 0.75494 & 0.50250 & 0.06928 & 1.000 & 1.000 & 1a \\
\hline $187 \mathrm{Ti}$ & Ti58 & 0.75509 & 0.75281 & 0.18526 & 1.000 & 1.000 & 1a \\
\hline $188 \mathrm{Ti}$ & Ti59 & 0.75642 & 0.50272 & 0.30628 & 1.000 & 1.000 & 1a \\
\hline $189 \mathrm{Ti}$ & Ti60 & 0.75527 & 0.75225 & 0.42140 & 1.000 & 1.000 & 1a \\
\hline $190 \mathrm{Ti}$ & Ti61 & 0.87994 & 0.75250 & 0.06928 & 1.000 & 1.000 & 1a \\
\hline $191 \mathrm{Ti}$ & Ti62 & 0.88187 & 0.50247 & 0.18887 & 1.000 & 1.000 & 1a \\
\hline $192 \mathrm{Ti}$ & Ti63 & 0.88158 & 0.75370 & 0.30340 & 1.000 & 1.000 & 1a \\
\hline $193 \mathrm{Ti}$ & Ti64 & 0.88323 & 0.50273 & 0.43833 & 1.000 & 1.000 & 1a \\
\hline
\end{tabular}

